\title{
1 Influence of the conditions of sensitization on the characteristics of $p$-DSCs 2 sensitized with asymmetric squaraines
}

*Corresponding authors: claudia.barolo@unito.it; danilo.dini@uniroma1.it

Running title: DSCs of $p$-type with asymmetric squaraines as sensitizers

\begin{abstract}
The effect of the conditions of sensitization on the photoelectrochemical performance of $p$-type dye-sensitized solar cells ( $p$-DSCs) with screen-printed nickel oxide $(\mathrm{NiO})$ photocathodes is analysed. The dye-sensitizers employed in the present study are asymmetric squaraines. The conditions of sensitization differ for the relative concentration of the anti-aggregating agent CDCA (chenideoxycholic acid) with respect to the concentration of the dye-sensitizer. The co-adsorption of CDCA onto $\mathrm{NiO}$ electrode brings about a decrease in the surface concentration of the anchored dye as well as a blue shift of the characteristic wavelengths of optical absorption of the asymmetric squaraines considered here. Beside this, the employment of CDCA as co-adsorbent reduces the overall conversion performance of the resulting squaraine-sensitized $p$-DSCs with consequent diminution of the short-circuit current density. This result is ascribed to the acid action of CDCA towards the amminic nitrogen of the squaraines. Quantum efficiency spectra show that CDCA acts as a quencher of the intrinsic photoelectrochemical activity of NiO. Moreover, CDCA does not interfere with the mechanism of charge injection effectuated by the photoexcited squaraines. The photoelectrochemical impedance spectra was analysed employing a model of equivalent circuit developed for semiconducting nanostructure electrodes.
\end{abstract}

KEYWORDS: squaraine, dye-sensitized solar cell, p-type, nickel oxide 


\section{INTRODUCTION}

The success of the dye-sensitized solar cell (DSC) [1] as an alternative device for the conversion of solar energy is due to a variety of reasons which include the use of low cost materials and the adoption of scalable methods utilizing experimental apparatuses that are non-sophisticated and economically practicable [2]. The operating principle of the photoelectrochemical DSC [3] is based on the process of charge photoinjection from the excited state of a light-absorbing molecule (the dye-sensitizer) [4] to a semiconducting electrode. In a DSC the starting event of charge separation in the dyesensitizer is followed by the simultaneous injection of charges with a given sign to the semiconducting substrate (on which the dye-sensitizer has been immobilized), and the accompanying injection of charges of opposite sign to an opportune constituent of the redox couple. The redox couple thus acts as a shuttle of electrons (or redox mediator) in the electrolyte of the DSC. Moreover, the shuttle warrants the passage of the current through the electrolytic phase [5]. Electrode sensitization with strong light-absorbers is a process necessary to enlarge the spectral window of photoelectrochemical activity of the semiconducting electrode with respect to the situation in which the semiconductor is in the bare, pristine state. This is because the unmodified inorganic semiconductors are typically wide-bandgap materials with $E_{\mathrm{g}}>3 \mathrm{eV}$, i.e. absorb energies not inferior to the near UV threshold [6]. Beside high optical absorbance in the visible range, the dyesensitizer must fulfil the matching of the frontier energy levels with the band edges of the semiconductor [7]. Such a phenomenon of proper energy level alignment is necessary to accomplish the charge transfer to/from the semiconducting substrate with monodirectional, irreversible, efficient and kinetically/thermodynamically favourable features [8]. The optically excited dye can inject either an electron or a hole to the semiconducting substrate: in the first case one refers to $n$ type DSCs [9] while the second case of charge transfer occurs during operation of $p$-type photoelectrochemical cells [10]. The DSC with tandem configuration ( $t$-DSC) is also conceivable provided both electrodes of the $t$-DSC are dye-sensitized and, consequently, display photoelectrochemical activity [11-14]. In such a configuration the $t$-DSCs have a higher theoretical limit of maximum efficiency of solar energy conversion (above 40\%) with respect to $n$ - and $p$-DSCs as predicted by the thermodynamic calculations of Shockley and Queisser in the early sixties [15]. In the realization of $t$-DSC the bottleneck is represented by the lack of matching of the photocurrents carried by the best performing photoanode and the best performing photocathode when these are combined together in the same photoelectrochemical cell. In fact, photocathodes (or $p$-type semiconductors) hardly reach photocurrent densities larger than $5 \mathrm{~mA} \mathrm{~cm}{ }^{-2}$ in the corresponding $p$-DSCs [10], whereas photoanodes (or $n$-type semiconductors) can produce photocurrent densities as high as $17.5 \mathrm{~mA} \mathrm{~cm}^{-}$ ${ }^{2}$, i.e. at least 3 times larger [9]. The problem of the general amelioration of $p$-DSC performance has been tackled by following several directions of research. These embrace the improvement of the $p$-type semiconducting electrode, namely mesoporous $\mathrm{NiO}[16-33,33]$, the alteration of the nature of the electrolyte and the optimization of its composition [10, 34$36]$ and the design and the realization of better-performing dye-sensitizers [22, 37-40]. In particular, we considered the aspect of the development of new dye-sensitizers [41] based on the structure of the squaraine [42] for the improvement of NiO-based $p$-DSCs. There are several reasons for adopting squaraines as dye-sensitizers of DSCs [43-49]: i) versatility of their synthetic chemistry [44-46], ii) strong optical absorption in the region of the near infrared (NIR) [50-54] and consequent absorption with complementary features with respect to traditional organic/organometallic dye-sensitizers [7], and iii) within the class of squaraines for $p$-DSCs [30, 55-58], the existence of several examples displaying HOMO energy level lower than the upper edge of the semiconductor valence band, and having the LUMO energy level higher than the level of the redox shuttle at the condition of equilibrium. In a previous work [41] we have demonstrated the suitability of a series of asymmetric squaraines (Figure 1), denominated DS_45 and DS_47, as dyes sensitizers of NiO cathodes for the assembly of $p$-DSCs. In that recent study we also showed that DS_45 produced photoelectrochemical cells with larger conversion efficiency in comparison to the benchmark sensitizer pSQ2 (0.043 vs 0.038) [56] when screen-printed NiO [32] was employed as the nanoporous cathode and no anti-aggregating agent [59] was used in the solution of sensitization.
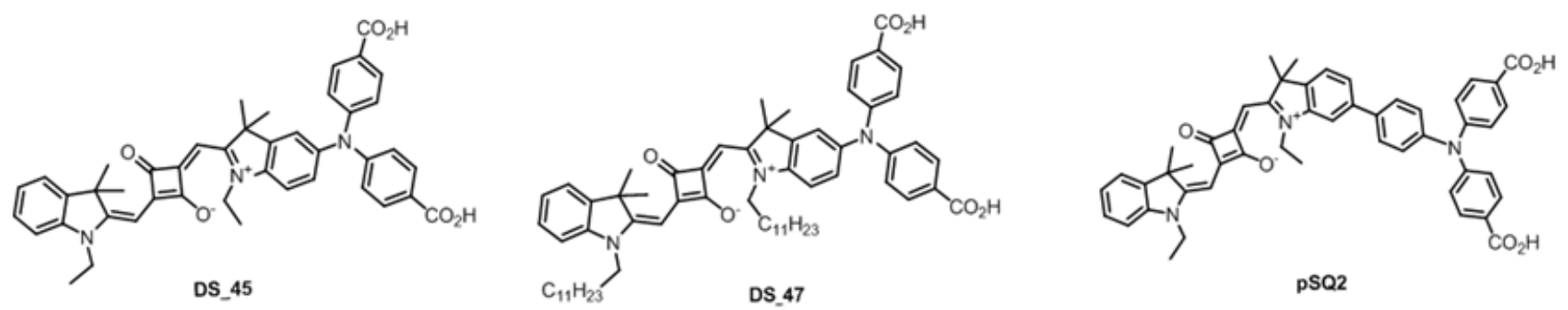

Figure 1. Structures of the three asymmetric squaraines DS_45, DS_47 and the benchmark pSQ2 considered here as sensitizers of $p$-DSCs.

The use of a co-adsorbent [60-62] in the solution of sensitization with squarainic dyes is a common practice [55] for preventing the formation of aggregates of $H$ - or $J$-type on the surface of the sensitized electrode. The risk of aggregate formation is higher for molecules having a flat structure, scarce conformational freedom and relatively large sizes as in the case of squaraines like those displayed in figure 1 . The existence of such surface aggregates is generally deleterious for the photoactivated charge transfer between the optically excited dye and the electrode surface [63,64] although this statement can lose validity for some classes of dye-sensitizers $[65,66]$. Therefore, it is expected that the addition of an anti- 
aggregating agent like CDCA (Figure 2) [67, 68] in the sensitization solutions of $\mathrm{NiO}$ cathodes influences to some extent the performance of the corresponding photoelectrochemical cells of $p$-type when the squaraines shown in figure 1 are employed as dye-sensitizers. DS_45 and DS_47 (Figure 1) differ for the length of the alkyl substituent on the nitrogen atom of the condensed pyrrolic ring. Such a structural difference can affect the extent of dye-loading [69] as well as the probability of having intermolecular aggregation between the immobilized molecules of the dye-sensitizer [70]. The present work deals with the characterization of the $p$-DSCs adopting DS_45, DS_47 and the benchmark pSQ2 as photocathode sensitizers when the sensitization of the electrode is conducted in the presence of CDCA as anti-aggregating co-adsorbent.

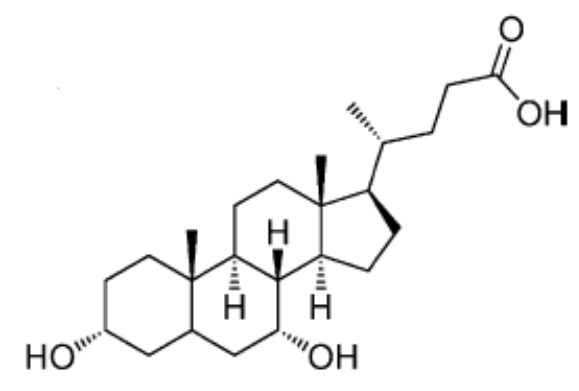

\section{CDCA}

Figure 2. Structure of the co-adsorbent CDCA (chenodeoxycholic acid) employed here as an anti-aggregating agent against the formation of H-aggregates on the surface of the squaraine-sensitized electrode.

In particular we present the $J V$ curves, incident photon-to-current conversion efficiency (IPCE) spectra and the spectra of photoelectrochemical impedance for the differently sensitized cells in order to rationalize the influence of the co-adsorbent CDCA (Figure 2) on the overall performance of the $p$-DSCs sensitized with the squaraines shown in figure 1 . We have considered screen-printed $\mathrm{NiO}$ [30] in the configuration of thin film (thickness $l<4 \mu \mathrm{m}$ ) [71] as mesoporous cathodes of the $p$-DSCs analyzed here.

\section{MATERIALS AND METHODS}

\section{Syntheses of squaraines}

The synthetic procedures for the realization of the squaraines DS_45 and DS_47 (Figure 1) are detailed in [41] and references therein. The synthetic path of DS_45 and DS_47 is common for both dyes and follows the same sequence of reactions (Scheme 1) [41]. The scheme consists of several steps with the alkylated derivative of 5-bromoindole (4) representing the precursor for the formation of the squaraine skeleton (Scheme 1). The benchmark dye pSQ2 (Figure 1) was homemade according to a slightly modified version of the procedure described in [56]. All the commercial reagents and solvents used in the preparation of squaraines DS_45 and DS_47 were purchased either from Sigma-Aldrich or from Fluka and were used as received.

\section{Preparation and deposition of $\mathrm{NiO}$ electrodes}

For the screen-printing deposition of $\mathrm{NiO}$ electrodes we first prepared a precursor paste of viscous consistency, which contained preformed nanoparticles of $\mathrm{NiO}$ (diameter $\leq 50 \mathrm{~nm}$ ). The details of the chemical composition of the paste are given in [55] and [41]. All the chemicals employed in the preparation of the precursor paste for the screen-printing procedure were of the highest degree of purity available and were purchased from Sigma-Aldrich and Fluka. The chemicals were used without any further procedure of purification. The details regarding the preparation of the precursor paste, its modality of screen-printing deposition onto fluorine-doped tin oxide (FTO)-covered glass substrates (from Solaronix) and the sintering conditions are given in [32]. The resulting screen-printed $\mathrm{NiO}$ electrode had a photoactive area of $0.25 \mathrm{~cm}^{2}$.

\section{NiO electrode sensitization}

The sensitization of screen-printed $\mathrm{NiO}$ photocathodes with the three squaraines shown in figure 1 was realized through electrode dipping in an ethanol solution containing $0.2 \mathrm{mM}$ dye-sensitizer and CDCA (Figure 2) at variable concentration. When CDCA was present the two concentration ratios were [CDCA]:[Dye] = 10:1 and 50:1. All screen-printed NiO samples were sensitized at room temperature for a couple of hours. Upon completion of the sensitization step the sensitized electrode was removed from the tincture solution and washed gently two times with the solvent of sensitization, i.e. pure ethanol. The optical transmittance of the squaraine-sensitized electrodes was measured using the double ray spectrometer 

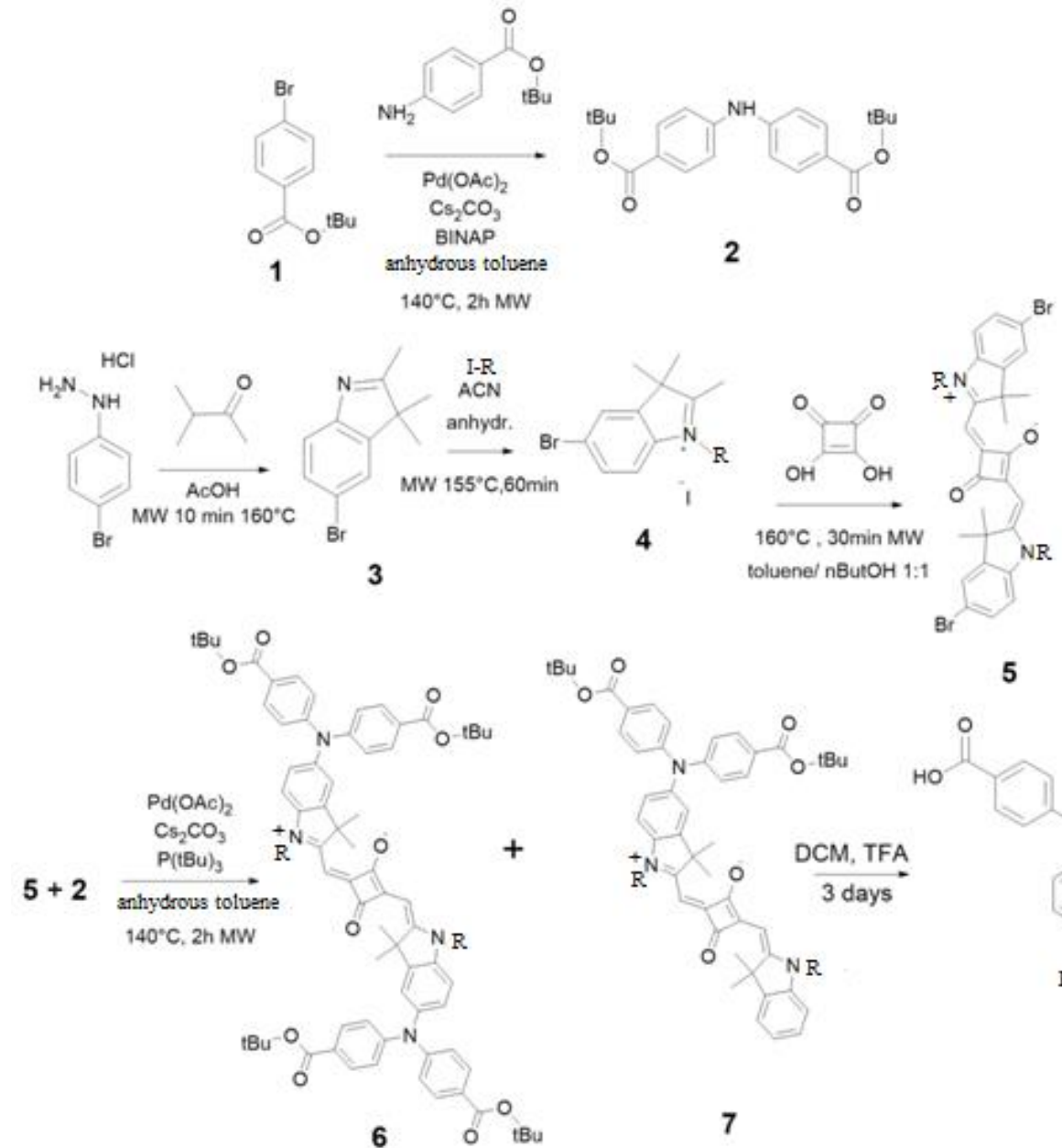

5

Scheme 1. Synthetic path adopted for the preparation of squaraines DS_45 $\left(\mathbf{8}, \mathrm{R}=-\mathrm{C}_{2} \mathrm{H}_{5}\right)$ and DS_47 $\left(\mathbf{8}, \mathrm{R}=-\mathrm{C}_{12} \mathrm{H}_{25}\right)$. In the intermediate step of $\mathrm{N}$-alkylation $\mathbf{3} \rightarrow \mathbf{4}$ the reactants ethyl-iodide and dodecyl-iodide were used for the attainment of DS_45 and DS_47, respectively.

\section{Counter electrode preparation}

The counter electrode of the $p$-DSC consisted of a substrate of FTO which was rendered electrocatalytically active upon platinization, i.e. upon formation of nanoisland of $\mathrm{Pt}$ on FTO. Platinised FTO was obtained through the procedure previously reported [72].

Assembly of the $\boldsymbol{p}$-DSC The screen-printed electrode of NiO and platinised FTO were assembled together in a sandwich configuration using the thermoplastic polymer Bynel ${ }^{\circledR}$ as spacer between the two electrodes and sealant of the cell. After electrodes sandwiching, the commercial liquid electrolyte HSE (high stability electrolyte) from Dyesol was injected inside the cell using the vacuum backfilling technique. HSE is based on the redox shuttle iodide/triiodide. The hole drilled on one glass substrate for electrolyte injection was finally sealed with the liquid resin 3035B (from Threebond) that gets hardened upon ultra-violet (UV) curing.

\section{Photoelectrochemical cell characterization}

The $J V$ curves of the $p$-DSCs were recorded under illumination using the solar simulator Solar Test 1200 KHS (class B) at $1000 \mathrm{~W} \mathrm{~m}^{-2}$. The artificial sun employed here had the emission spectrum AM 1.5 G. The IPCE curves were recorded using a computer-controlled set-up consisting of a Xe lamp (Mod.70612, Newport) coupled to a monochromator (Cornerstone 130 from Newport), and a Keithley 2420 light-source meter. The spectra of electrochemical impedance were determined using an AUTOLAB PGSTAT12® from Metrohm at the condition of open circuit potential in both dark conditions and under solar simulator illumination. For the experiments of electrochemical impedance spectroscopy (EIS) conducted under illumination the applied potential was also modulated within $\pm 20 \mathrm{mV}$ with respect to the value of open circuit. In the EIS experiments conducted in the absence of illumination the potential perturbation had an amplitude of $10^{-2} \mathrm{~V}$. The frequency 
range of the impedance perturbation varied depending on the cell state, i.e. if illuminated or not. Impedance spectra were recorded within the frequency ranges $10^{-1}-10^{5} \mathrm{~Hz}$ and $10^{-2}-10^{5} \mathrm{~Hz}$ when the cell was under illumination and in dark conditions, respectively. The impedance spectra were modeled and fitted using Z-View software from Scribner Associates Inc.

\section{RESULTS AND DISCUSSION}

\section{Optical properties of the sensitized NiO electrodes}

The optical transmission properties of the NiO films sensitized with DS_45, DS_47 and pSQ2 (Figure 1) when the coadsorbent concentration varied in the sensitizing solution are presented in figure 3 . Within the visible and NIR ranges the transmittance values of sensitized $\mathrm{NiO}$ resulted systematically lower than those of bare $\mathrm{NiO}$. This result clearly evidences the sensitizing action of the three squaraines under consideration on screen-printed $\mathrm{NiO}$ and constitutes the necessary starting point to achieve photocathodes suitable for the applications of $p$ - and $t$-DSCs. Another significant aspect of the observed trend in the transmission spectra in figure 3 is related to the extent of the diminution of electrode transmittance upon variation of CDCA concentration in the solution of sensitization. The larger ratio of concentrations [CDCA]/[dye] (= 50:1) in the tincturing solution leads to a weaker effect of $\mathrm{NiO}$ sensitization by the dye. This is expected as a consequence of the stronger tendency of anchoring for CDCA with respect to a squaraine sensitizer by virtue of the effect of mass [55]. Concomitant with the lower extent of sensitization is the smaller value of dye-loading for all the squarainic dye-sensitizers employed here (Table 1). The determination of dye-loading was conducted spectrophotometrically with the measurement of the optical spectra of the solution of the dye molecules desorbed from the NiO electrode surface. Dye-sensitizer desorption is achieved quantitatively by immersing the sensitized electrode in a solution of acetic anhydride for 5 minutes [55]. The data in table 1 indicate that the increase in the amount of CDCA in the dipping solution causes a blue-shift of the wavelength of maximum optical absorption $\left(\lambda_{\mathrm{MAX}}\right)$. This fact could not be noticed in a previous work from us which reported the effect of CDCA on the sensitizing action of symmetric squaraines [55]. In the present case we ascribe such an effect of absorption shift to the protonation of the nitrogen of the triphenylamminic units in the structures presented in figure 1. This process of protonation can cause a modification of the extent of electronic conjugation in the aromatic species with consequences on the allowed energies of absorption. As previously outlined, the higher the concentration of CDCA in the sensitizing solution, the higher the transmittance values and, consequently, the lower the amount of anchored dye (Table 1). The decrease of transmission is quite evident for all three dyes when the concentration ratio [CDCA]:[dye] passes from 0:1 to 10:1. Upon further increase in the ratio [CDCA]:[dye] to 50:1 only the DS series did not produce any sensible decrease of transmittance. This is true especially for DS_45 (Figure 3, middle frame).

Unlike the process of sensitization with DS squaraines, the presence of a relatively large amount of CDCA in the dipping solution impedes the anchoring of pSQ2 almost completely since the spectrum of $\mathrm{NiO}$ immersed in the solution with $[C D C A]:[\mathrm{pSQ} 2]=50: 1$ practically retraces the one of bare $\mathrm{NiO}$ (Figure 3, top frame). Such a specific behavior of pSQ2 is caused by the structural differences between pSQ2 and the DS compounds (Figure 1) with pSQ2 having a larger skeleton and then a slower kinetics of anchoring with respect to DS_45 and DS_47. In the presence of CDCA the considerably different lengths of the alkyl chains in DS_45 and DS_47 has no apparent effect on the control of the process of dye anchoring (Table 1). This conclusion is somewhat in contrast to what was previously determined by the analysis of NiO sensitization process using symmetric squaraines [55].

Table 1. Variations in the wavelength of maximum absorption $\left(\lambda_{\operatorname{MAX}}\right)$ and of the corresponding value of optical transmission $(T)$ of squaraine-sensitized $\mathrm{NiO}$ (thickness, $l=2 \mu \mathrm{m}$ ) upon variation of the composition of the sensitization solution (data extracted from figure 3). The changes in the composition of the sensitization solution are expressed in terms of the concentration ratio $[\mathrm{CDCA}] /[$ dye $]$ with the squarainic dye-sensitizers having a fixed concentration ([dye $]=0.2 \mathrm{mM}$ ). In the last column on the right the surface concentration (in $\mathrm{mol} \mathrm{cm}^{-2}$ ) of the dye loaded by screen-printed $\mathrm{NiO}$ is reported.

\begin{tabular}{ccccc}
\hline Dye & $\begin{array}{c}\text { CDCA:dye } \\
\text { molar ratio }\end{array}$ & $\lambda_{\text {MAX }} / \mathrm{nm}$ & $T / \%$ & $\begin{array}{c}\text { Dye loading }\left(* 10^{-8}\right. \\
\left.\text { mol } / \mathrm{cm}^{2}\right)\end{array}$ \\
\hline pSQ2 & pure & $\mathbf{6 0 5}$ & $\mathbf{5 1 . 6 7}$ & $\mathbf{1 . 2 8} \pm \mathbf{0 . 1 2}$ \\
& $\mathbf{1 0 : 1}$ & $\mathbf{6 0 9}$ & $\mathbf{5 5 . 0 7}$ & $\mathbf{0 . 9 6} \pm \mathbf{0 . 1 0}$ \\
DS_45 & $\mathbf{5 0 : 1}$ & $/$ & $/$ & $\mathbf{0 . 0 5} \pm \mathbf{0 . 0 3}$ \\
& pure & $\mathbf{6 4 7}$ & $\mathbf{5 3 . 3 1}$ & $\mathbf{1 . 5 2} \pm \mathbf{0 . 1 6}$ \\
& $\mathbf{1 0 : 1}$ & $\mathbf{6 3 5}$ & $\mathbf{5 9 . 0 6}$ & $\mathbf{0 . 6 8} \pm \mathbf{0 . 1 2}$ \\
DS_47 & $\mathbf{5 0 : 1}$ & $\mathbf{6 3 1}$ & $\mathbf{6 0 . 9 8}$ & $\mathbf{0 . 4 6} \pm \mathbf{0 . 0 9}$ \\
& pure & $\mathbf{6 4 7}$ & $\mathbf{4 8 . 4 1}$ & $\mathbf{1 . 1 2} \pm \mathbf{0 . 0 9}$ \\
& $\mathbf{1 0 : 1}$ & $\mathbf{6 6 0}$ & $\mathbf{5 3 . 6 1}$ & $\mathbf{0 . 8 5} \pm \mathbf{0 . 0 9}$ \\
\hline & $\mathbf{5 0 : 1}$ & $\mathbf{6 5 6}$ & $\mathbf{5 8 . 9 9}$ & $\mathbf{0 . 4 8} \pm \mathbf{0 . 0 8}$ \\
\hline
\end{tabular}



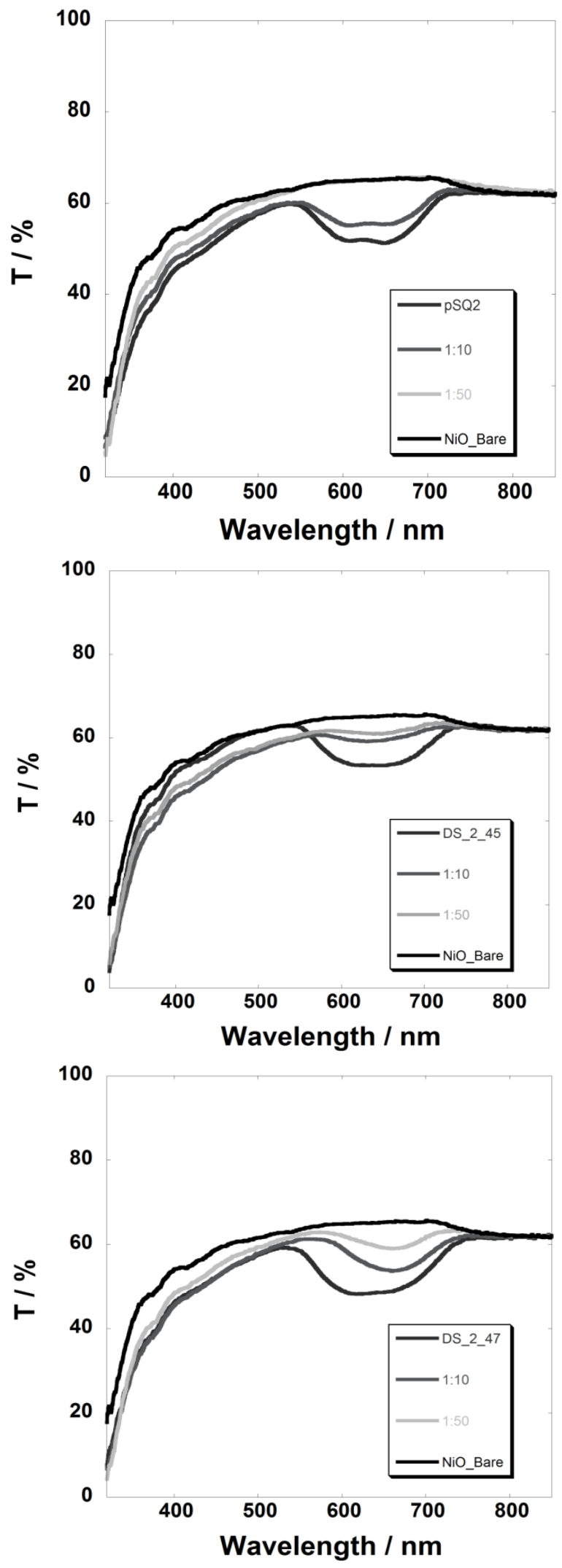

Figure 3. Spectral variations in optical transmittance of squaraine-sensitized NiO films when different concentrations of CDCA were used in the solution of sensitization. For the sake of comparison the spectrum of pristine NiO in the non sensitized state is also shown. The ratios in the legend indicate the molar ratio squaraine:CDCA. Top frame: spectra of pSQ2-sensitized NiO; middle frame: spectra of DS_45-sensitized NiO; bottom frame: DS_47-sensitized NiO. 

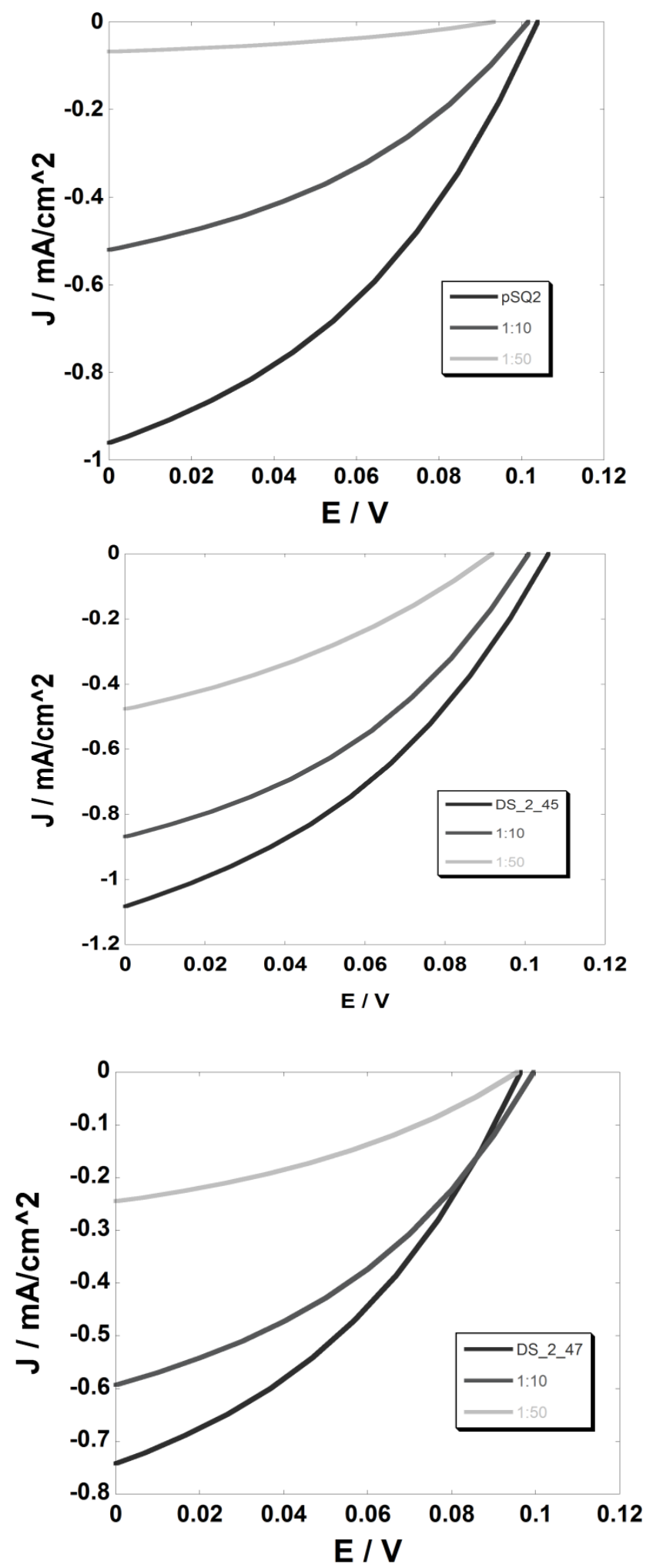

Figure 4. Characteristic $J V$ curves of the NiO-based $p$-DSCs with pSQ2 (top), DS_45 (middle) and DS_47 (bottom) as sensitizers. The profiles were determined when $\mathrm{NiO}$ photocathodes were sensitized at different values of [CDCA]. The concentration of the dye was kept constant. Black curves were determined when $\mathrm{NiO}$ cathode sensitization was conducted in the absence of CDCA ([dye]=0.2 $\mathrm{mM}$ ). The dark grey and light grey curves were determined when the molar ratio [dye]/[CDCA] in the solution of $\mathrm{NiO}$ sensitization were 1:10 and 1:50, respectively. 
Figure 4 shows the characteristic $J V$ curves of the $p$-DSCs derived from the differently sensitized NiO cathodes and table 2 reports the corresponding photoelectrochemical parameters of open circuit voltage $\left(V_{\mathrm{OC}}\right)$, short-circuit current density $\left(J_{\mathrm{SC}}\right)$, fill factor (FF) and overall conversion efficiency $(\eta)$ when the $p$-DSCs were illuminated with a sun simulator producing a radiation intensity of $1000 \mathrm{~W} \mathrm{~m}^{-2}$. In all cases examined here the effect of the presence of CDCA in the solution of $\mathrm{NiO}$ sensitization consists in the inhibition of the photoelectrochemical activity of the resulting photocathode when the dyes are the asymmetric squaraines DS_45, DS_47 and pSQ2 (Figure 1). The most affected parameter is a dynamic one, i.e. $J_{\mathrm{SC}}$, whereas the others tend to decrease more slowly upon increase in CDCA chemical activity in the solution of sensitization. This is somewhat unexpected since it is widely recognized that the co-adsorption of the anti-aggregating agent CDCA ameliorates the photoelectrochemical performance of squarine-sensitized photoelectrodes by virtue of the kinetic stabilization which molecular isolation imparts to the actual photoexcited species that is immobilized on the electrode surface [48, 73-79]. The lack of any beneficial effect of CDCA co-adsorption on the $p$-DSC performances recorded here (Table 2) and the resulting detrimental influence could be due to three main reasons (existing either simultaneously or separately): i) the eventual absence of intermolecular aggregation between pSQ2, DS_45 and DS_47 molecules in the surface-immobilized state even when the electrode is sensitized in CDCA-free solutions since the sizes of the present series of squaraines (Figure 1) are large enough to avoid strong intermolecular interactions; ii) the inefficacy of the possible aggregates of DS_45, DS_47 and pSQ2 to retard or prevent the process of photoinduced electron transfer (et); and iii) the deleterious effect of squaraine protonation (due to co-adsorbed CDCA) on the photoactivation and/or realization of the et process due to electron trapping phenomena. The different structures of squaraines DS_45, DS_47 and pSQ2 (Figure 1) have no substantial effect on the extent of the loss in the overall performance as imparted by the co-sensitization with CDCA (Table 2). In the absence of the co-sensitizer, the most performing squaraine is DS_45 in accordance to previously reported results [41]. The external quantum efficiency determined at the condition of short-circuit presents spectral profiles (Figure 5) which are consistent with the data in table 2. The non-uniform diminution of spectral efficiency in correspondence of the characteristic absorption of squaraines DS_45, DS_47 and pSQ2 (Figure 1) [41] upon increase in the concentrations of CDCA (Figure 5) supports the hypothesis of an important effect of et quenching due to the dye protonation (point iii, vide supra) and deactivation of $\mathrm{NiO}$ photoactive sites rather than the exertion of an action of squaraine disaggregation by CDCA.

Table 2. Photoelectrochemical parameters of open circuit voltage ( $\left.V_{\mathrm{OC}}\right)$, short-circuit current density $\left(J_{\mathrm{SC}}\right)$, fill factor $(\mathrm{FF})$ and overall conversion efficiency $(\eta)$ of the $p$-DSCs displaying the characteristic $J V$ curves shown in figure 4.
Dye
[CDCA]:[dye]
$V_{\text {oc }} / \mathbf{m V}$
$J \mathrm{sc} / \mathbf{m A ~} \mathbf{c m}^{-2}$
FF / \%
$\eta / \%$

\begin{tabular}{cccccc}
\hline pSQ2 & $\mathbf{0 : 1}$ & $\mathbf{1 0 5}$ & $\mathbf{- 0 . 9 5 5}$ & $\mathbf{3 8 . 2}$ & $\mathbf{0 . 0 3 8}$ \\
& $\mathbf{1 0 : 1}$ & $\mathbf{1 0 3}$ & $\mathbf{- 0 . 5 1 5}$ & $\mathbf{3 8 . 1}$ & $\mathbf{0 . 0 2 0}$ \\
& $\mathbf{5 0 : 1}$ & $\mathbf{9 7}$ & $\mathbf{- 0 . 0 5 9}$ & $\mathbf{3 7 . 9}$ & $\mathbf{0 . 0 0 2}$ \\
DS_45 & $\mathbf{0 : 1}$ & $\mathbf{1 0 6}$ & $\mathbf{- 1 . 0 7 7}$ & $\mathbf{3 7 . 4}$ & $\mathbf{0 . 0 4 3}$ \\
& $\mathbf{1 0 : 1}$ & $\mathbf{1 0 2}$ & $\mathbf{- 0 . 8 6 1}$ & $\mathbf{3 8 . 3}$ & $\mathbf{0 . 0 3 3}$ \\
& $\mathbf{5 0 : 1}$ & $\mathbf{9 6}$ & $\mathbf{- 0 . 4 5 0}$ & $\mathbf{3 3 . 7}$ & $\mathbf{0 . 0 1 4}$ \\
DS_47 & $\mathbf{0 : 1}$ & $\mathbf{1 0 1}$ & $\mathbf{- 0 . 7 0 8}$ & $\mathbf{3 7 . 1}$ & $\mathbf{0 . 0 2 7}$ \\
& $\mathbf{1 0 : 1}$ & $\mathbf{1 0 0}$ & $\mathbf{- 0 . 5 8 7}$ & $\mathbf{3 8 . 1}$ & $\mathbf{0 . 0 2 2}$ \\
& $\mathbf{5 0 : 1}$ & $\mathbf{9 6}$ & $\mathbf{- 0 . 2 4 0}$ & $\mathbf{3 6 . 0}$ & $\mathbf{0 . 0 0 8}$ \\
\hline
\end{tabular}

The IPCE (incident photon-to-current conversion efficiency) spectra are characterized by the presence of two different contributions: the most intense one at lower wavelengths is due to bare $\mathrm{NiO}$, whereas the minor one at higher wavelengths is attributable to the photoactivity of the dye. The two ranges of photoelectrochemical activity of squaraine-sensitized pDSCs are complementary to each other and both of them contribute to the overall efficiency of conversion. An interesting aspect of the spectra shown in figure 5 is the extent of IPCE diminution which is caused by CDCA co-sensitization in correspondence to the wavelength range $300-540 \mathrm{~nm}$ when the squaraine varies its structure. As previously stated, within this particular interval bare $\mathrm{NiO}$ displays its intrinsic photoelectrochemical activity [30, 55, 80]. Moreover, it is known that CDCA deactivates the photoelectroactivity of the bare electrodes of the NiO samples prepared via screen-printing [55]. The comparative analysis of the trends of IPCE spectra (Figure 5) reveals that the extent of $\mathrm{NiO}$ photoelectrochemical deactivation caused by CDCA co-adsorption is influenced by the nature of the dye-sensitizer. Since the deactivation of the intrinsic photoelectrochemical activity of $\mathrm{NiO}$ is directly proportional to the amount of adsorbed CDCA we conclude here that the concentration of chemisorbed CDCA increases in the adopted conditions of sensitization on going from DS_45 to pSQ2 through DS_47. This trend is then correlated to the variations of dye-loading evaluated on freshly sensitized NiO electrodes according to the data summarized in table 1 . These facts further confirm the inhibitory action of the disaggregating acidic agent CDCA towards the photoelectrochemically active sites of $\mathrm{NiO}$. 

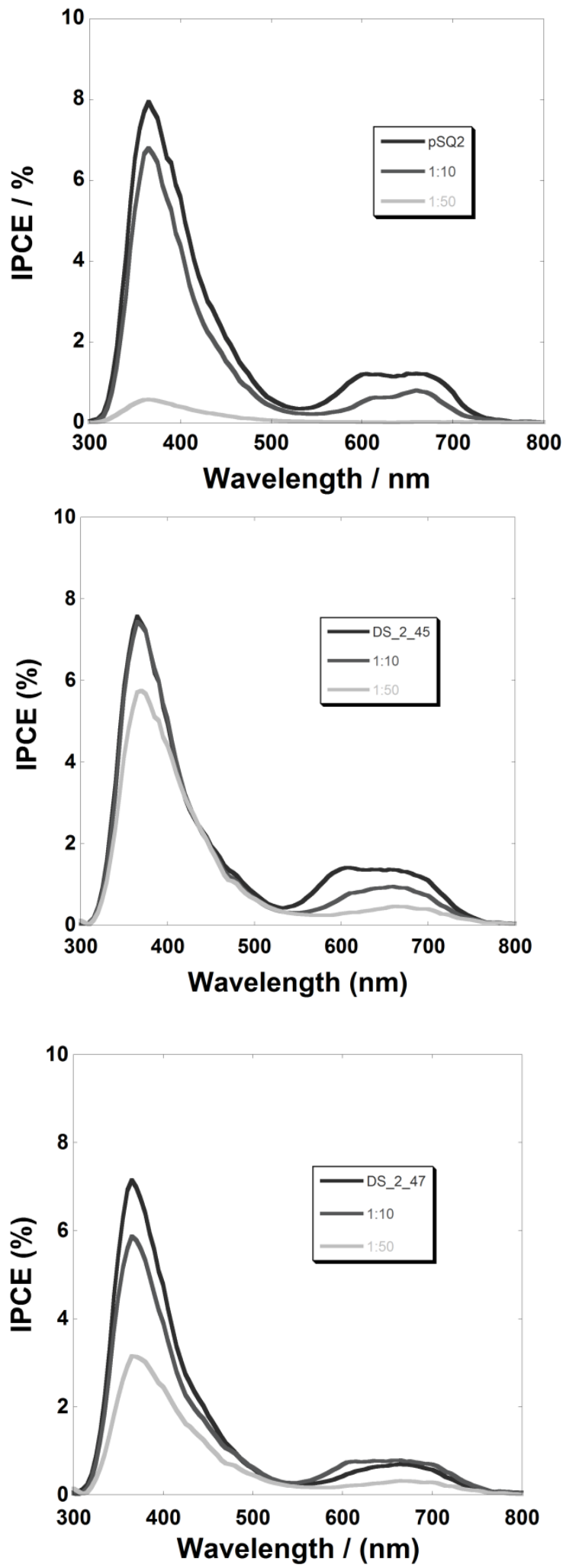

were illuminated and maintained at the potential of open circuit (Table 2).

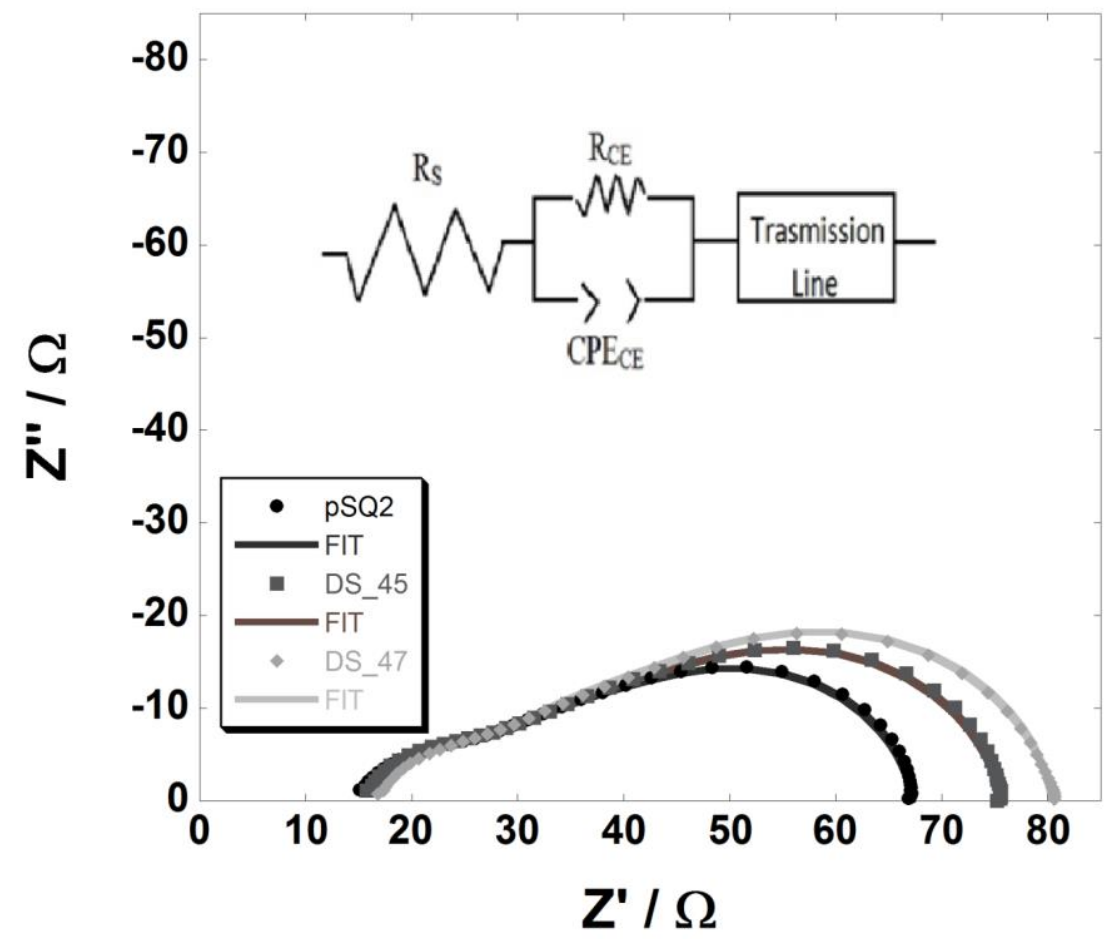

Figure 6. Nyquist plots of the electrochemical impedance spectra of the $p$-DSC sensitized with pSQ2 (black dots), DS_45 (dark grey squares) and DS_47 (light grey diamonds) at their respective values of open circuit photopotential (see table 2). The spectra have been recorded when CDCA was not employed in the sensitization solution. The inset represents the equivalent circuit adopted to fit all the photoelectrochemical impedance spectra of the differently squaraine-sensitized $p$ DSCs.

For the fitting of the photoelectrochemical impedance data shown in figures 6 and 7 we adopted the equivalent circuit shown in the inset of figure 6 . This circuit was developed originally by Bisquert for the analysis of the impedance response given by the $n$-type version of the DSCs [81]. The characteristics of the frequency response given by the sole photocathode are delineated in the transmission line (inset of figure 6) [82, 83], whereas $R_{\mathrm{s}}, R_{\mathrm{CE}}$ and CPE $\mathrm{CE}_{\text {(a constant phase element) }}$ refer to the characteristics of the DSC electrolyte (namely $\left.R_{\mathrm{s}}\right)$ and of the counter electrode $\left(R_{\mathrm{CE}}\right.$ and CPE $\left.\mathrm{CE}_{\mathrm{CE}}\right)$. The circuital element of the transmission line includes all the electrical parameters associated to the charge transport properties and the capacitive characteristics of the sensitized electrode when this is constituted by an electroactive nanoporous material $[82,83]$. The transmission line comprises the resistive contributions $R_{\mathrm{t}}$ (the charge transport resistance through the photocathode), $R_{\text {rec }}$ (the charge recombination resistance at the photocathode/electrolyte interface) [30, 55-57, 80] and the capacitive element $C_{\mu}$ [81]. The capacitive element of chemical capacitance [84, 85] is expressed in $\mathrm{F} \mathrm{m}^{-3}$ and describes the capability of exchanging charge carriers per unit volume upon variation of the chemical potential of the charge carriers $\mu_{\mathrm{i}}$ (in $\mathrm{J}$ ). The parameter $\mu_{\mathrm{i}}$ is controlled by the density of charge carriers $N_{\mathrm{i}}$ (in $\mathrm{m}^{-3}$ ) within the working electrode [86]. In an electrodic system like the squaraine-sensitized cathode of $\mathrm{NiO}$ the charge carrier is the hole, the chemical potential of which is altered by the variation in the photopotential caused by the changes in light intensity [87]. The chemical capacitance is related to the charge storage properties of the photoelectrode under different conditions of photocarrier injection [88]. Equations 1-4 [83] correlate the electrical terms of $R_{\mathrm{t}}, R_{\mathrm{rec}}$ and $C_{\mu}$ to the charge carrier (holes) parameters $\tau_{\mathrm{d}}$, $\tau_{\mathrm{h}}, D_{\mathrm{h}}$ and $L_{\mathrm{h}}$ :

$$
\begin{aligned}
& \tau_{\mathrm{d}}=R_{\mathrm{t}} * C_{\mu} \\
& \tau_{\mathrm{h}}=R_{\mathrm{rec}} * C_{\mu} \\
& D_{\mathrm{h}}=L_{\mathrm{h}}{ }^{2} / \tau_{\mathrm{d}}=L_{\mathrm{h}}{ }^{2} /\left(R_{\mathrm{t}} * C_{\mu}\right) \\
& L_{\mathrm{h}}=l_{*} *\left(R_{\mathrm{rec}} / R_{\mathrm{t}}\right)^{1 / 2}
\end{aligned}
$$



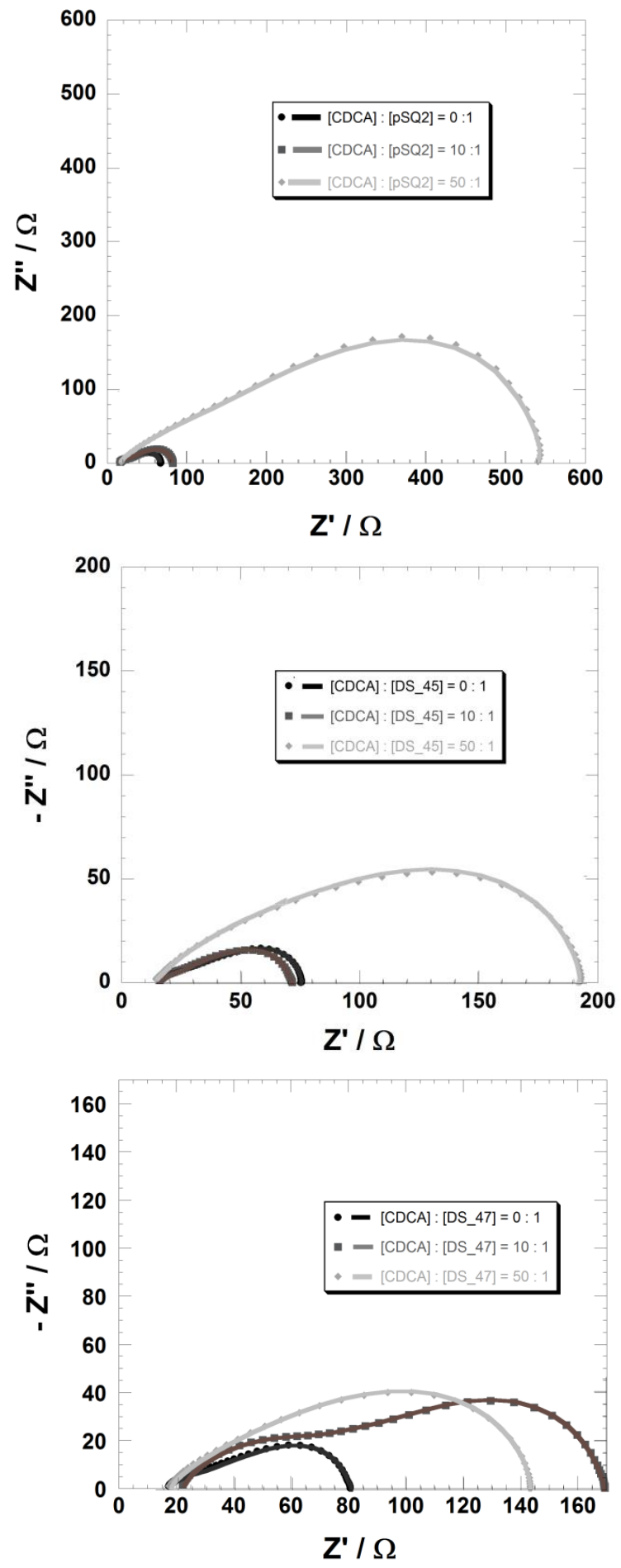

Figure 7. Nyquist plots of the electrochemical impedance spectra of the $p$-DSCs sensitized with pSQ2 (top), DS_45 (middle) and DS_47 (bottom) at their respective values of open circuit photopotential under different conditions of NiO sensitization (see table 2). Black circles and black line indicate the impedance spectra and the fitting curve, respectively, obtained from the equivalent circuit presented in figure 6 . when the sensitizing solution does not contain CDCA. Dark grey squares and light grey full line indicate the impedance spectra and the corresponding fit, respectively, when solution sensitization has the relative composition $[\mathrm{CDCA}]:[$ squaraine] $=10: 1$. Light grey diamonds and light grey full line indicate the impedance spectra and the corresponding fit, respectively, when solution sensitization has the composition [CDCA]:[squaraine] $=50: 1$.

In eqs. 1-4 the microscopic parameters of $\tau_{\mathrm{d}}, \tau_{\mathrm{h}}, D_{\mathrm{h}}$ and $L_{\mathrm{h}}$ refer to the time of hole diffusion, the average lifetime of the hole, the diffusion coefficient of the hole and the length of hole diffusion, respectively [89]. In eq.4, $l$ represents the nominal thickness of the electrode as determined by a profilometer [32]. It must pointed out that the porous nature and the non uniform, inhomogeneous character of the screen-printed $\mathrm{NiO}$ electrode $[30,32,71]$ considered here does not allow the correct evaluation of the length of the path actually available to the charge carriers simply through film thickness 
determination. The distance charge carriers can actually traverse is expected to be larger than the nominal value of film thickness, i.e. $2 \mu \mathrm{m}$. Table 3 lists the fitting values of the three main electrical parameters associated to the transport and capacitive properties of the $\mathrm{NiO}$ photocathode when it is sensitized in solutions with varying concentrations of the coadsorbent CDCA. The values in table 3 were utilized to fit the photoelectrochemical impedance data shown in figures 6 and 7.

Table 3. Fitting values of the electrical parameters related to the charge transport properties $R_{\mathrm{t}}$ and $R_{\mathrm{rec}}$ of the squarainesensitized $\mathrm{NiO}$ cathode. The chemical capacitance $C_{\mu}$ is also reported. The three electrical terms are included in the element of transmission line (see inset of figure 6). The transmission line is the circuital element employed to describe the impedance of the photoelectrochemical cell at the photopotential of open circuit. The set of parameters reported here allowed the interpolation of the corresponding impedance spectra reported in figures 6 and 7 . The quality of the fitting was evaluated using the $\chi^{2}$-test $\left(<10^{-5}\right.$ except for the fitting of the spectrum of the $p$-DSC sensitized with pSQ2 when the sensitization conditions were $[\mathrm{CDCA}]:[\mathrm{dye}]=50: 1)$.

\begin{tabular}{ccccc}
\hline Dye & {$[$ CDCA] :[dye $]$} & $R_{\mathrm{t}} / \Omega$ & $R_{\mathrm{rec}} / \Omega$ & $C_{\mu} / \mu \mathrm{F}$ \\
\hline pSQ2 & $\mathbf{0 : 1}$ & $\mathbf{2 1 . 2} \pm \mathbf{1 . 3}$ & $\mathbf{8 0 . 4} \pm \mathbf{4 . 0}$ & $\mathbf{4 5} \pm \mathbf{5}$ \\
& $\mathbf{1 0 : 1}$ & $\mathbf{2 4 . 0} \pm \mathbf{1 . 3}$ & $\mathbf{1 1 7 . 3} \pm \mathbf{4 . 6}$ & $\mathbf{4 3} \pm \mathbf{4}$ \\
& $\mathbf{5 0 : 1}$ & $\mathbf{3 1 2 . 4} \pm \mathbf{7 . 9}$ & $\mathbf{6 6 5 . 0} \pm \mathbf{1 2 . 1}$ & $\mathbf{3 9} \pm \mathbf{2}$ \\
DS_45 & $\mathbf{0 : 1}$ & $\mathbf{2 4 . 6} \pm \mathbf{1 . 6}$ & $\mathbf{9 6 . 1} \pm \mathbf{4 . 8}$ & $\mathbf{4 0} \pm \mathbf{1}$ \\
& $\mathbf{1 0 : 1}$ & $\mathbf{2 5 . 9} \pm \mathbf{1 . 5}$ & $\mathbf{9 4 . 2} \pm \mathbf{5 . 1}$ & $\mathbf{4 0} \pm \mathbf{2}$ \\
& $\mathbf{5 0 : 1}$ & $\mathbf{7 4 . 7} \pm \mathbf{2 . 3}$ & $\mathbf{1 3 3 . 0} \pm \mathbf{7 . 0}$ & $\mathbf{3 8} \pm \mathbf{2}$ \\
DS_47 & $\mathbf{0 : 1}$ & $\mathbf{2 4 . 6} \pm \mathbf{1 . 3}$ & $\mathbf{1 1 1 . 7} \pm \mathbf{4 . 2}$ & $\mathbf{3 9} \pm \mathbf{1}$ \\
& $\mathbf{1 0 : 1}$ & $\mathbf{2 9 . 4} \pm \mathbf{1 . 6}$ & $\mathbf{9 1 . 8} \pm \mathbf{4 . 0}$ & $\mathbf{3 9} \pm \mathbf{1}$ \\
& $\mathbf{5 0 : 1}$ & $\mathbf{5 5 . 6} \pm \mathbf{2 . 3}$ & $\mathbf{2 0 1 . 4} \pm \mathbf{5 . 8}$ & $\mathbf{5 0} \pm \mathbf{4}$ \\
\hline
\end{tabular}

Table 4 presents the values calculated through eqs. 1-4 for the four main charge carrier parameters that describe the diffusive properties and time-stability characteristics of the holes photoinjected in the differently sensitized $\mathrm{NiO}$ photocathodes. Data tabulated in tables 3 and 4 present some common trends for the three differently sensitized photocathodes of NiO: a) general tendency of the NiO resistive terms $R_{\mathrm{t}}$ and $R_{\text {rec }}$ to increase upon increase in the CDCA concentration in the sensitizing solution; b) increase in the characteristic times $\tau_{\mathrm{d}}$ and $\tau_{\mathrm{h}}$ for the photoinjected holes upon increase in the CDCA concentration in the sensitizing solution; c) general tendency of the diffusive parameters $D_{\mathrm{h}}$ and $L_{\mathrm{h}}$ to decrease upon increase in the CDCA concentration in the sensitizing solution; d) scarce dependence of the chemical capacitance $C_{\mu}$ on the concentration of CDCA in the solution of sensitization.

Table 4. Values of the parameters related to the characteristics of charge carriers $\tau_{\mathrm{d}}, \tau_{\mathrm{h}}, D_{\mathrm{h}}$ and $L_{\mathrm{h}}$. This set of data was calculated via eqs.1-4 utilizing the fitting data reported in table 3.

\begin{tabular}{cccccc}
\hline Dye & {$[$ CDCA] $:[$ dye $]$} & $\tau_{\mathrm{d}} / \mathbf{m s}$ & $\tau_{\mathrm{h}} / \mathbf{m s}$ & $D_{\mathbf{h}} / \mathbf{1 0}^{-\mathbf{6}} \mathbf{c m}^{2} \mathbf{s}^{-1}$ & $\boldsymbol{L}_{\mathbf{h}} / \boldsymbol{\mu m}$ \\
\hline pSQ2 & $\mathbf{0 : 1}$ & $\mathbf{0 . 9 7} \pm \mathbf{0 . 1 0}$ & $\mathbf{3 . 6 5} \pm \mathbf{0 . 1 2}$ & $\mathbf{1 5 8 . 4} \pm \mathbf{1 4 . 2}$ & $\mathbf{3 . 6 2} \pm \mathbf{0 . 4 2}$ \\
& $\mathbf{1 0 : 1}$ & $\mathbf{1 . 0 3} \pm \mathbf{0 . 1 1}$ & $\mathbf{5 . 0 4} \pm \mathbf{0 . 2 0}$ & $\mathbf{1 8 9 . 8} \pm \mathbf{1 6 . 2}$ & $\mathbf{4 . 4 2} \pm \mathbf{0 . 2 1}$ \\
& $\mathbf{5 0 : 1}$ & $\mathbf{1 2 . 2} \pm \mathbf{0 . 8}$ & $\mathbf{2 5 . 9} \pm \mathbf{0 . 9 6}$ & $\mathbf{6 . 9 8} \pm \mathbf{0 . 9 8}$ & $\mathbf{2 . 9 2} \pm \mathbf{0 . 2 0}$ \\
DS_45 & $\mathbf{0 : 1}$ & $\mathbf{0 . 9 8} \pm \mathbf{0 . 0 8}$ & $\mathbf{3 . 9 5} \pm \mathbf{0 . 4 6}$ & $\mathbf{1 5 9 . 2} \pm \mathbf{1 5 . 4}$ & $\mathbf{3 . 8 5} \pm \mathbf{0 . 2 4}$ \\
& $\mathbf{1 0 : 1}$ & $\mathbf{1 . 0 4} \pm \mathbf{0 . 0 6}$ & $\mathbf{3 . 7 7} \pm \mathbf{0 . 5 2}$ & $\mathbf{1 3 9 . 6} \pm \mathbf{1 0 . 1}$ & $\mathbf{3 . 8 1} \pm \mathbf{0 5 0}$ \\
& $\mathbf{5 0 : 1}$ & $\mathbf{2 . 7 6} \pm \mathbf{0 . 1 2}$ & $\mathbf{4 . 9 4} \pm \mathbf{0 . 6 5}$ & $\mathbf{2 5 . 8} \pm \mathbf{8 . 2}$ & $\mathbf{2 . 6 7} \pm \mathbf{0 . 8 2}$ \\
DS_47 & $\mathbf{0 : 1}$ & $\mathbf{0 . 9 6} \pm \mathbf{0 . 0 5}$ & $\mathbf{4 . 2 6} \pm \mathbf{0 . 1 9}$ & $\mathbf{1 8 1 . 8} \pm \mathbf{5 . 2}$ & $\mathbf{4 . 3 6} \pm \mathbf{0 . 1 6}$ \\
& $\mathbf{1 0 : 1}$ & $\mathbf{1 . 1 5} \pm \mathbf{0 . 0 8}$ & $\mathbf{3 . 5 8} \pm \mathbf{0 . 2 2}$ & $\mathbf{1 0 8 . 3} \pm \mathbf{6 . 3}$ & $\mathbf{3 . 5 3} \pm \mathbf{0 . 4 6}$ \\
& $\mathbf{5 0 : 1}$ & $\mathbf{2 . 7 8} \pm \mathbf{0 . 3 2}$ & $\mathbf{1 0 . 0} \pm \mathbf{1 . 0 2}$ & $\mathbf{5 2 . 2} \pm \mathbf{4 . 0}$ & $\mathbf{3 . 8 1} \pm \mathbf{0 . 6 2}$ \\
\hline
\end{tabular}

The observation in point (a) is ascribable to the diminution of photoinjected charge carriers upon increase in the concentration of the co-adsorbed CDCA and the concomitant diminution of chemisorbed dye-sensitizer (see dye-loading values in table 1) due to the recognized action of photoelectrochemical passivation exerted specifically on NiO by surfaceimmobilized CDCA (Figure 5) [55]. A decrease in the diffusive time and hole lifetime [point (b)] is consistent with the diminution of the concentration of photoinjected charges if the mechanism of charge transport through a mesoporous semiconductor like screen-printed $\mathrm{NiO}$ occurs via trap-mediated hopping between confined states having a fixed concentration within the nanostructured system [90]. The generalized decrease in the two directly correlated hole transport parameters $D_{\mathrm{h}}$ and, to a lesser extent, $L_{\mathrm{h}}$ (Eqs. 3 and 4) with the increasing concentration of the surface-immobilized CDCA 
[point (c)] is not so straightforward since these two parameters are mainly associable to the bulk properties of the metal oxide electrode rather than its surface composition. A tentative explanation for such an observation could be the occurrence of charge-trapping phenomena exerted by the co-adsorbed CDCA, in addition to the effect of surface passivation aforementioned. Under these circumstances we suppose that chemisorbed CDCA induces an effect of immobilization towards the photoinjected holes due to the presence of the excess of negative charge following its deprotonation (the latter reaction is necessary to start the process of surface immobilization of CDCA onto $\mathrm{NiO}$
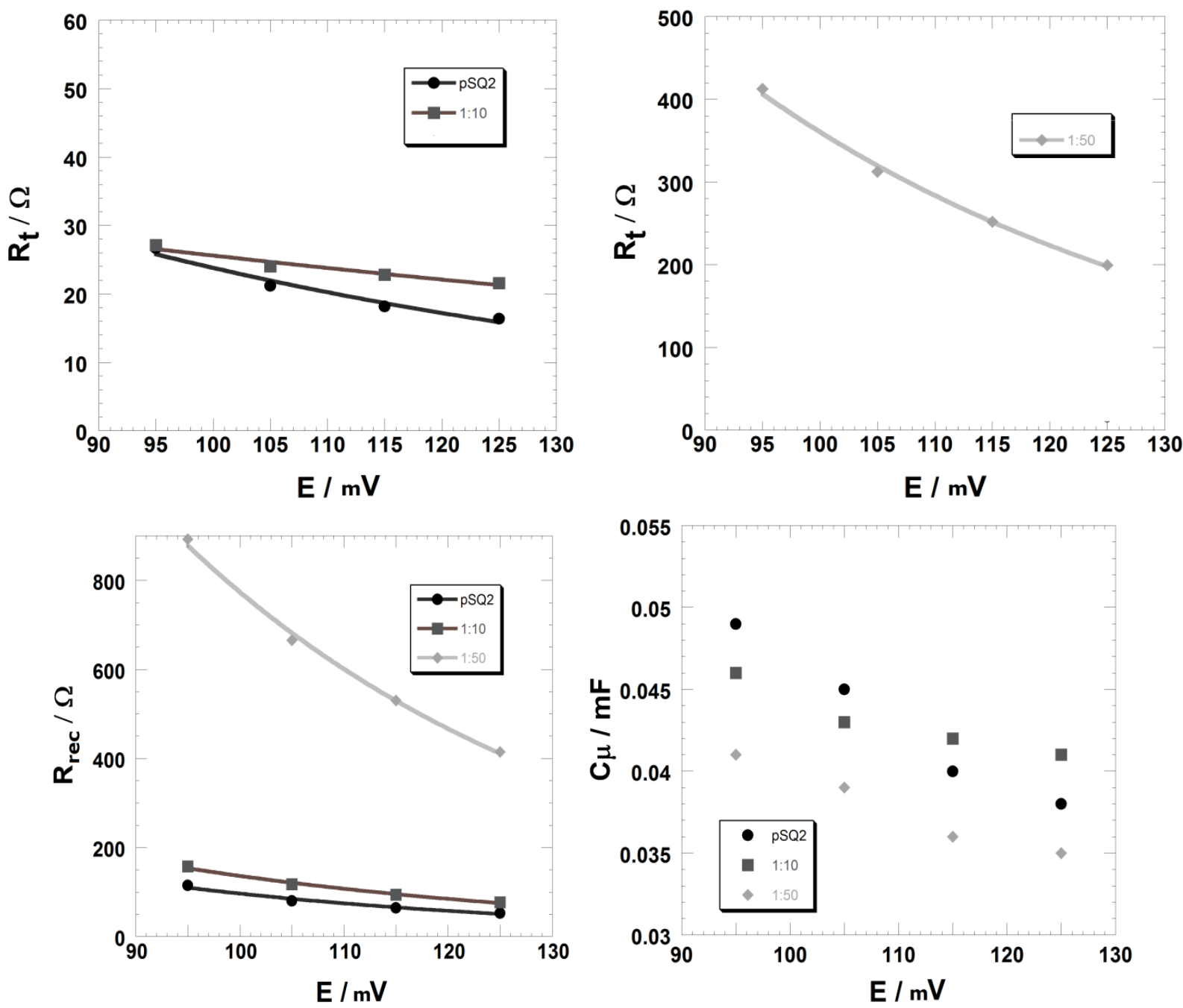

Figure 8. Potential dependence of the circuital parameters $R_{\mathrm{t}}$ (top), $R_{\mathrm{rec}}$ (middle) and $C_{\mu}$ (bottom) defining the transmission line of the equivalent circuit shown in figure 6. Sensitizer: pSQ2. The co-sensitization of the NiO electrodes was conducted in solutions with concentration ratios [CDCA]:[pSQ2] = 0:1 (black circles), 10:1 (dark grey squares) and 50:1 (light grey diamonds).

). Since screen-printed $\mathrm{NiO}$ is nanostructured $[30,32,71]$, i.e. possesses a large surface arealeffective volume ratio, the phenomenon of charge trapping from surface-confined species is expected to have a non negligible importance since it involves a relatively large portion of the system with respect to its wholeness. As far as the observation in point (d) is concerned, i.e. the scarce dependence of the chemical capacitance $C_{\mu}$ on the concentration of CDCA in the solution of sensitization, the lack of a univocal trend in the chemical capacitance $C_{\mu}$ upon variation in the CDCA concentration in the sensitizing solution (Table 3 ) is consistent with the fact that the chemical capacitance is mostly affected by the nature of the electrode material itself rather than the nature of the chemisorbed species [41]. In fact, the variation in the concentration of CDCA in the solution of sensitization affects the amount of CDCA chemisorbed onto the NiO electrode surface but not the chemical capacitance $C_{\mu}$ of the electrode itself. Since capacitance is the ratio of the charge distribution $\mathrm{Q}$ at an interface to the potential variation $\Delta \mathrm{V}$ that such distribution develops at the same interface, we expect that a poor/extensive charge photoinjection will be correspondingly accompanied by a small/large gradient of interfacial potential with the resulting quasi-constancy of the ratio $\mathrm{Q} / \Delta \mathrm{V}$. With these premises the parameter $C_{\mu}$ can be considered as poorly dependent on the 
extent of charge photoinjection if the nature of the electrode is kept invariant and only the nature of the dye-sensitizer is modified. We also recorded the impedance spectra of the $p$-DSCs under steady-state conditions of applied potential within the range $V_{\mathrm{OC}} \pm 20 \mathrm{mV}$ (not shown). This additional series of measurements was carried out in order to determine the changes in the electrical parameters of the photocathode when the operative conditions of the photoelectrochemical cell were varied. The potential dependence of the fitting circuital parameters $R_{\mathrm{t}}, R_{\mathrm{rec}}$ and $C_{\mu}$, and the corresponding variations in the microscopic properties $\tau_{\mathrm{d}}, \tau_{\mathrm{h}}, D_{\mathrm{h}}$ and $L_{\mathrm{h}}$ for the various photocathodes are presented in figures 8-13.
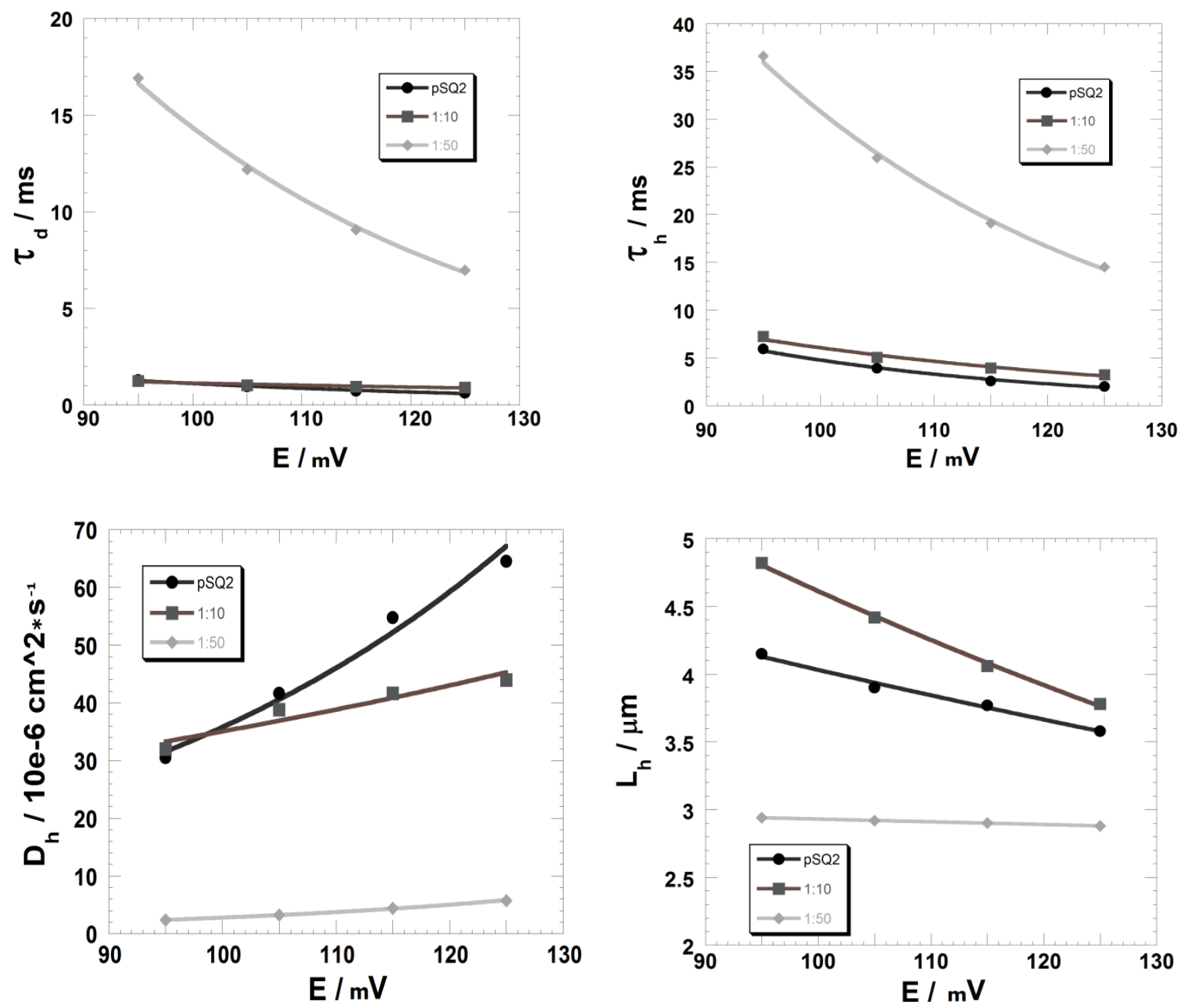

Figure 9. Potential dependence of the microscopic properties $\tau_{\mathrm{d}}$ (top left), $\tau_{\mathrm{h}}$ (top right), $D_{\mathrm{h}}$ (bottom left) and $L_{\mathrm{h}}$ (bottom right) related to the holes photoinjected in pSQ2-sensitized $\mathrm{NiO}$. These hole parameters were calculated from the values of $R_{\mathrm{t}}, R_{\text {rec }}$ and $C_{\mu}$ reported in figure 8 using eqs. 1-4. Conditions of sensitization: [CDCA]:[pSQ2] = 0:1 (black circles), 10:1 (dark grey squares) and 50:1 (light grey diamonds). 

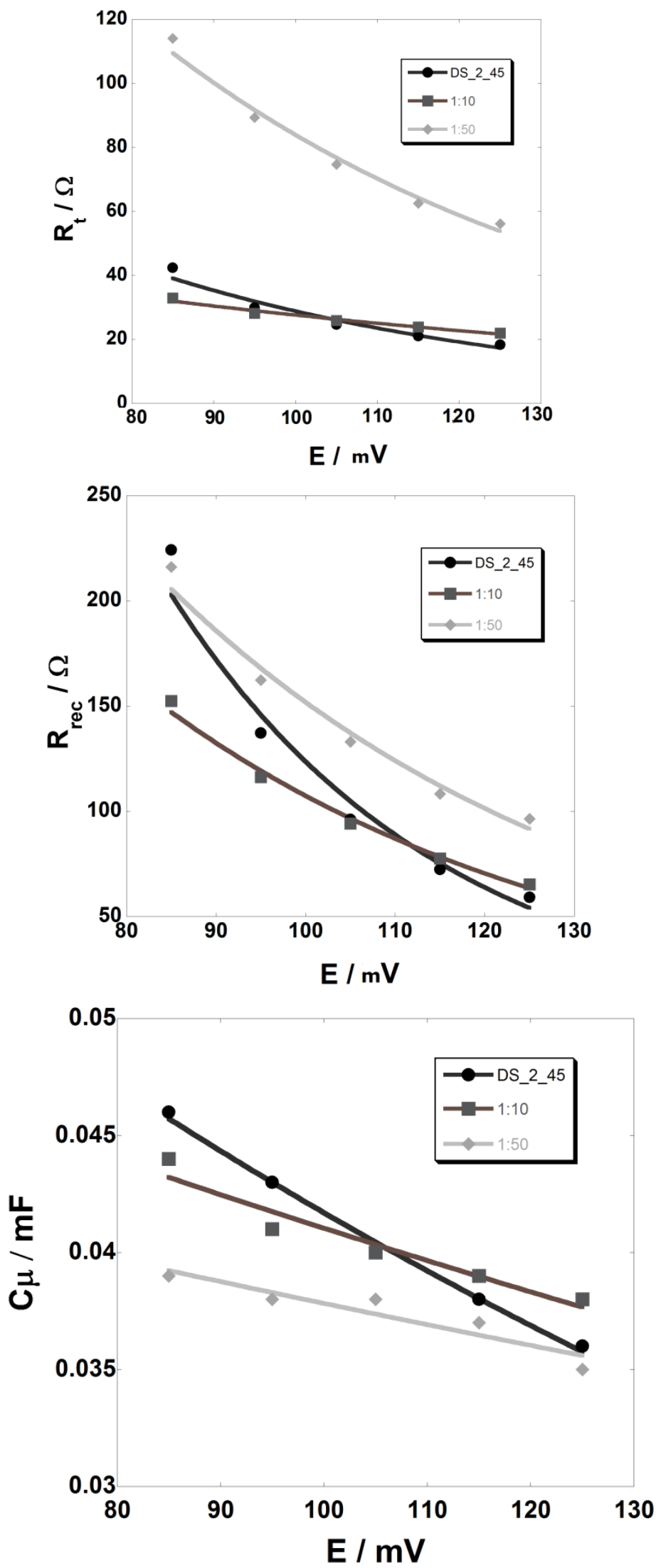

Figure 10. Potential dependence of the circuital parameters $R_{\mathrm{t}}$ (top), $R_{\text {rec }}$ (middle) and $C_{\mu}$ (bottom) defining the transmission line of the equivalent circuit shown in figure 6. Sensitizer: DS_45. The co-sensitization of the NiO electrodes was 

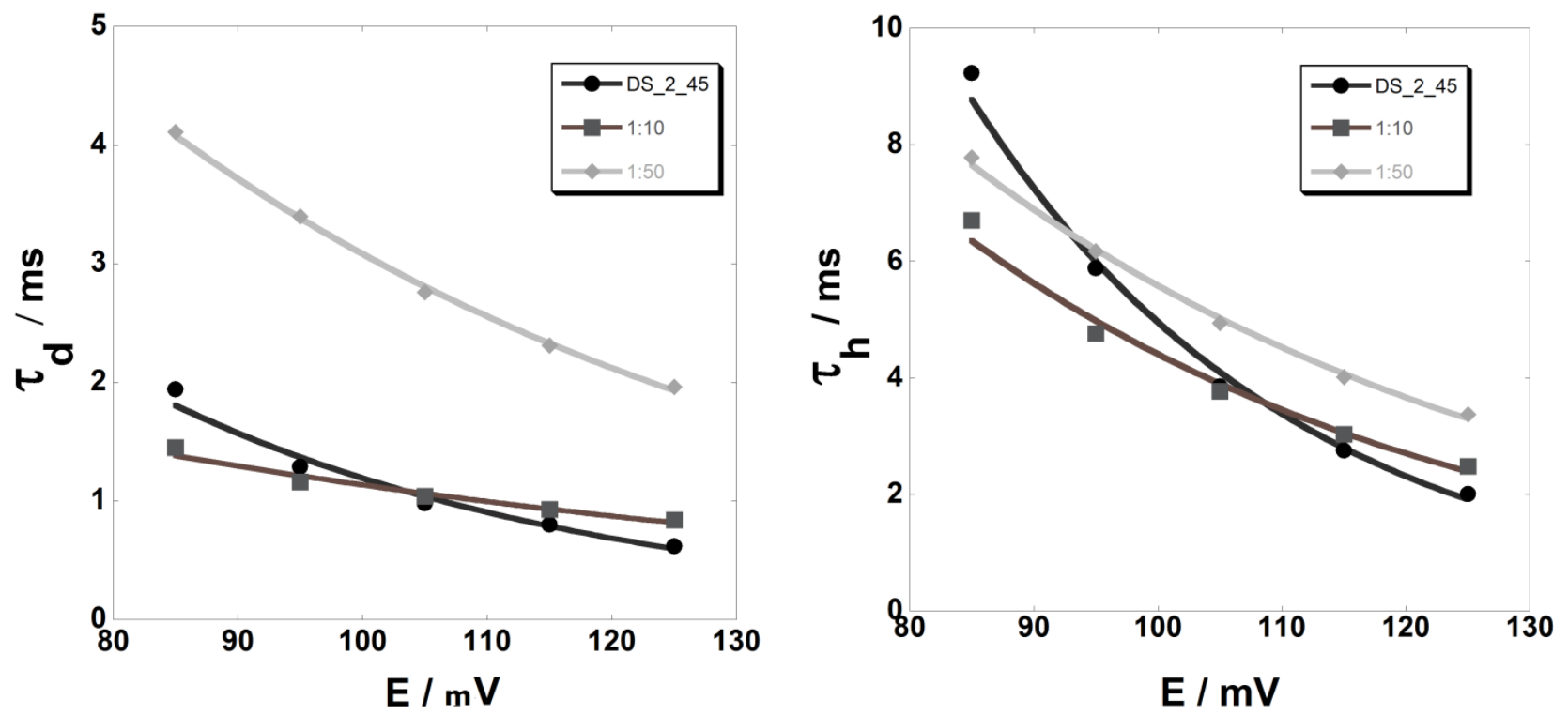

479
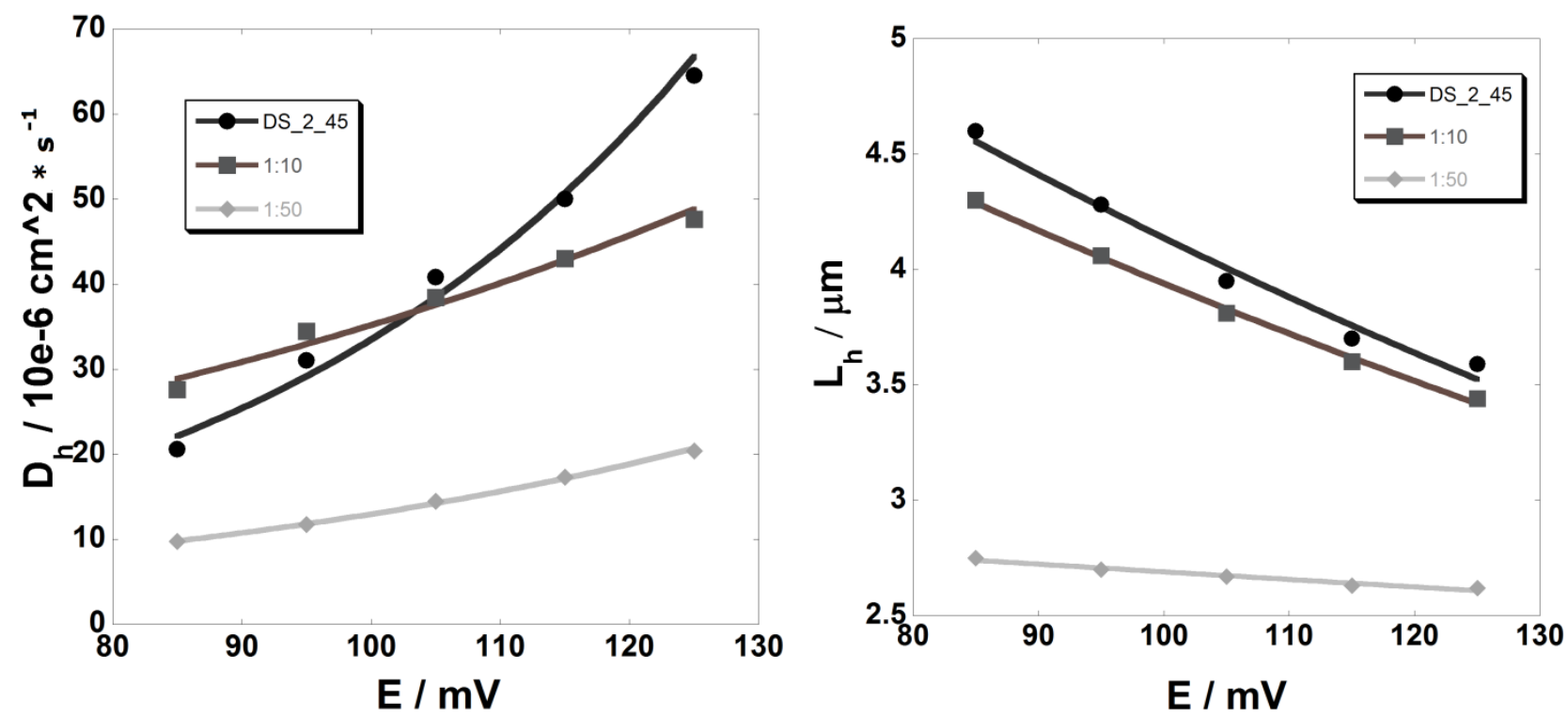

Figure 11. Potential dependence of the microscopic properties $\tau_{\mathrm{d}}$ (top left ), $\tau_{\mathrm{h}}$ (top right), $D_{\mathrm{h}}$ (bottom left) and $L_{\mathrm{h}}$ (bottom right) related to the holes photoinjected in DS_45-sensitized NiO. These hole parameters were calculated from the values of $R_{\mathrm{t}}, R_{\mathrm{rec}}$ and $C_{\mu}$ reported in figure 10 using eqs. 1-4. Conditions of sensitization: [CDCA]:[DS_45] = 0:1 (black circles), 10:1 (dark grey squares) and 50:1 (light grey diamonds). 

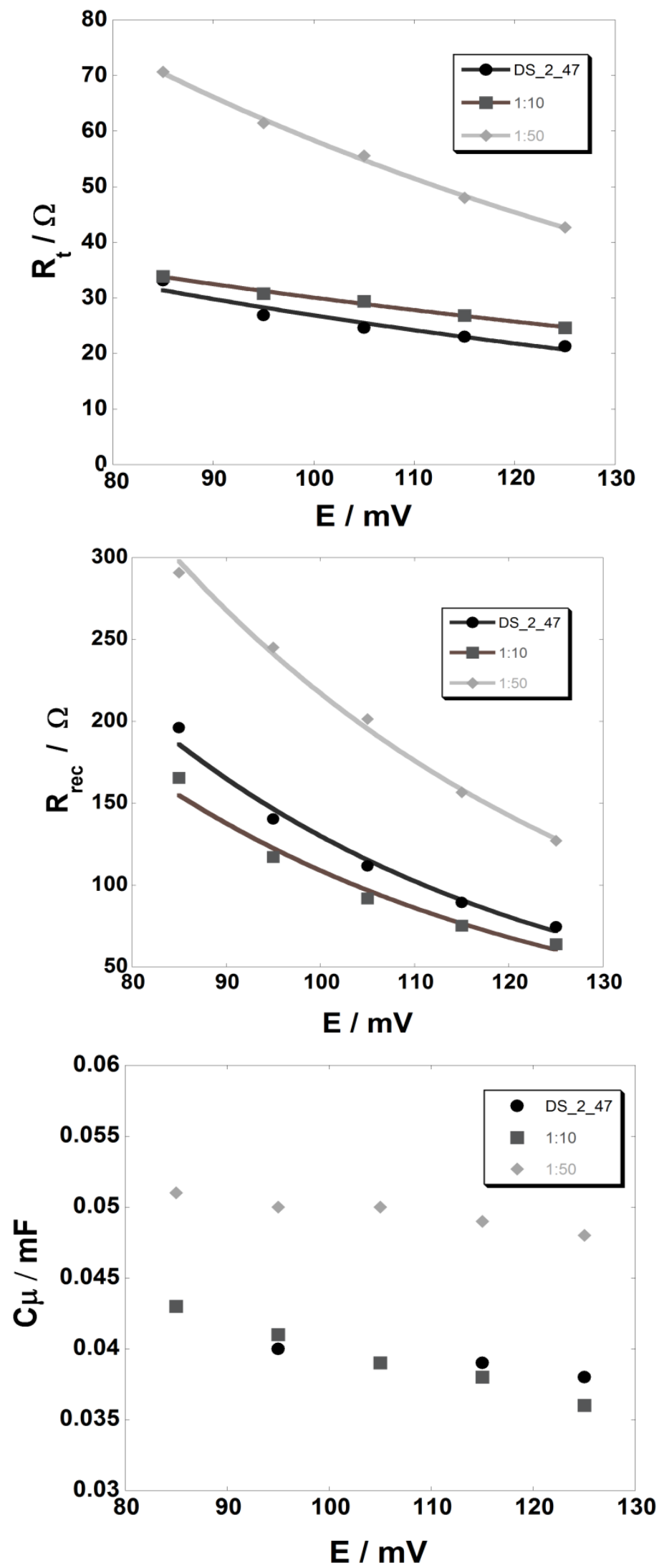

Figure 12. Potential dependence of the circuital parameters $R_{\mathrm{t}}$ (top), $R_{\mathrm{rec}}$ (middle) and $C_{\mu}$ (bottom) defining the transmission line of the equivalent circuit shown in figure 6. Sensitizer: DS_47. The co-sensitization of the NiO electrodes was conducted in solutions with concentration ratios [CDCA]:[DS_47] = 0:1 (black circles), 10:1 (dark grey squares) and 50:1 

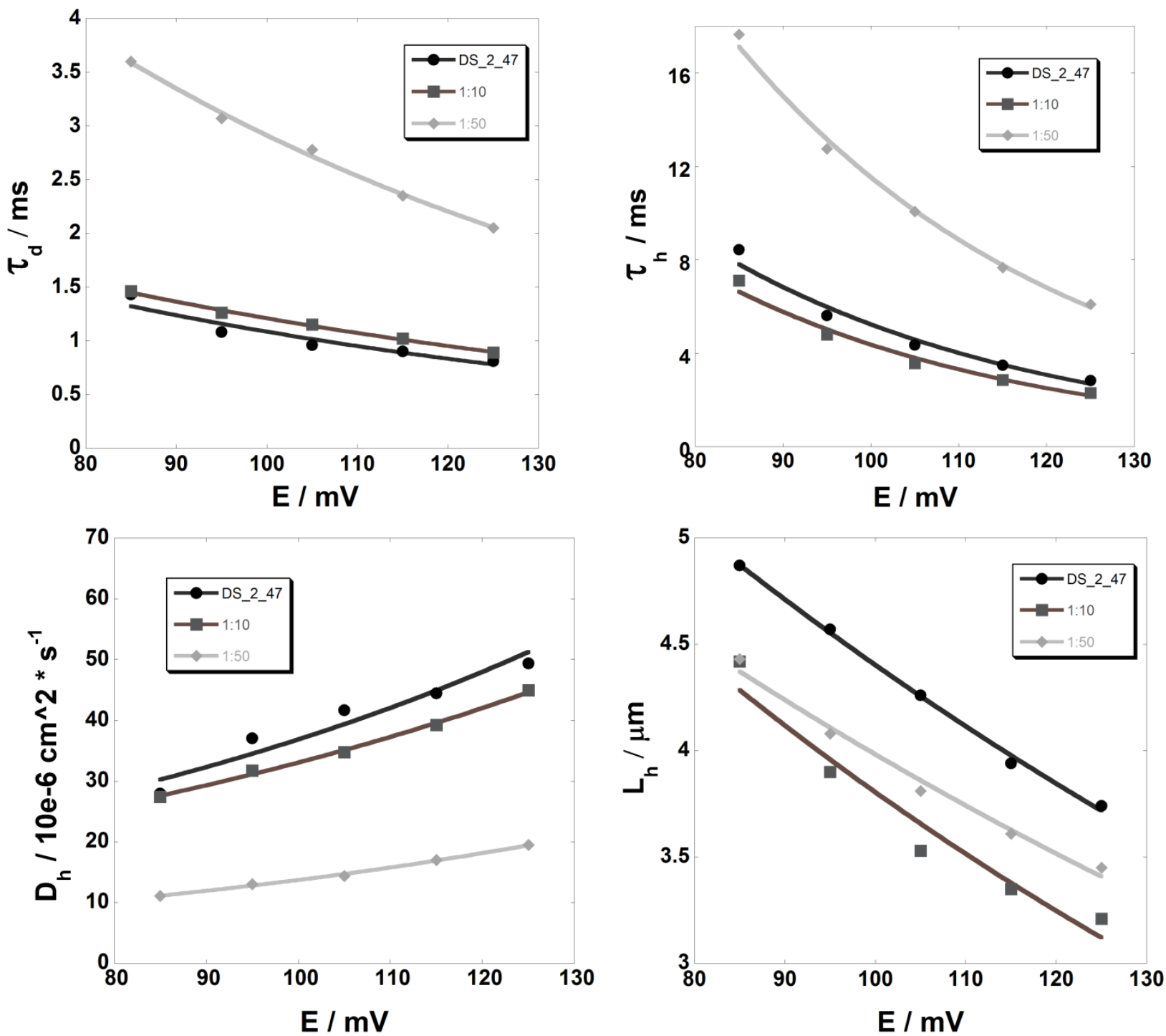

Figure 13. Potential dependence of the microscopic properties $\tau_{\mathrm{d}}$ (top left), $\tau_{\mathrm{h}}$ (top right), $D_{\mathrm{h}}$ (bottom left) and $L_{\mathrm{h}}$ (bottom right) related to the holes photoinjected in DS_47-sensitized NiO. These hole parameters were calculated from the values of $R_{\mathrm{t}}, R_{\mathrm{rec}}$ and $C_{\mu}$ reported in figure 12 using eqs. 1-4. Conditions of sensitization: [CDCA]:[DS_47] = 0:1 (black circles), 10:1 (dark grey squares) and 50:1 (light grey diamonds).

On going from the range $E_{\text {appl }}<V_{\mathrm{oc}}$ to the range $E_{\text {appl }}>V_{\mathrm{oc}}$ the $p$-DSC passes from a regime of reverse bias to the one of forward bias when the photoelectrochemical cell is illuminated. Therefore, the general augmentation of the applied potential is expected to provoke the photoinjection of an increased amount of charge carriers (the holes). Such a photoeffect diminishes the resistive terms $R_{\mathrm{t}}$ and $R_{\text {rec }}$ in all cases examined here (Figures 8,10 and 12, top and middle plots). This occurs by virtue of the increase in the concentration of mobile charge carriers when the oxide is forwardly polarized [91]. Moreover, the microscopic parameters of $\tau_{\mathrm{d}}, \tau_{\mathrm{h}}$ and $L_{\mathrm{h}}$ diminish upon increase in the forward potential and, consequently, upon the increase in the concentration of photocarriers. The decrease in these parameters with the concentration of mobile charge photocarriers is a consequence of the limited number of hopping sites [90] through which the photoinjected holes travel within the mesoporous structure of $\mathrm{NiO}$ before reaching the interface $\mathrm{FTO} / \mathrm{NiO}$ of charge collection. As expected, hole lifetime $\tau_{\mathrm{h}}$ also diminishes with the increase in the concentration of mobile charge carriers due to the occurrence of a recombination reaction between photoinjected holes and the iodide. The recombination reaction between photoinjected holes and the iodide is a process with bimolecular kinetics at the NiO/electrolyte interface. The rate of such a process is directly proportional to the concentration of the photoinjected charges $[26,55]$. The hole diffusion coefficient $D_{\mathrm{h}}$ decreases in the $p$-DSCs upon increase in CDCA concentration in the solution of $\mathrm{NiO}$ electrode sensitization. The decrease in $D_{\mathrm{h}}$ with $[C D C A]$ is verified within the whole range of applied potential for the $p$-DSCs sensitized with the three dyes (Figures 9, 11 
and 13, bottom left frames). This is in accordance with the fact that CDCA retards charge transport because of the exertion of a trapping effect on the photoinjected holes (vide supra). The latter phenomenon is then responsible for the lower hole diffusion coefficients determined in those photoelectrodes sensitized at the highest concentration of the co-adsorbent. With the exception of DS_47-sensitized cells, the two other asymmetric dyes produce cells that increase their respective hole diffusion coefficients upon forward bias, i.e. upon increase of the amount of injected charges. This constitutes an anomalous behaviour if charge transport occurs via a mechanism of trap-mediated hopping. The increase in charge carrier diffusivity in DS_45 and pSQ2-sensitized electrodes with the applied potential (Figures 9 and 11, bottom left plots) is associated to a generally scarce concentration of the charge carriers photoinjected in $\mathrm{NiO}$ after dye excitation and the presence of relatively large concentration gradients as well as longer diffusion lengths in these specific cases with respect to the other squaraine considered here.

\section{CONCLUSION}

The photoelectrochemical performance of variously sensitized $p$-DSCs with screen-printed NiO photocathodes was evaluated using the three asymmetric squaraines DS_45, DS_47 and pSQ2 as the dye sensitizers (Figure 1). In particular, we analysed the effect of the conditions of sensitization on the corresponding photoelectrochemical response of the cell when the solutions of sensitization contained variable concentrations of the co-adsorbent CDCA. In the absence of CDCA the overall efficiencies of the $p$-DSCs sensitized with DS_45, DS_47 and pSQ2 were dependent on the size of the alkyl substituent in the squaraine skeleton. Squaraine DS_45 produced the most efficient photoelectrochemical cells whereas the use of the sensitizer DS_47 led to less interesting performances. The co-adsorption of CDCA onto NiO electrode brings about a decrease in the surface concentration of the anchored dye as well as a blue shift of the characteristic wavelengths of optical absorption of the squaraines due to the protonation of the anchored dye and the consequent alteration of the extent of electronic conjugation in the surface immobilized dye. The adoption of CDCA as co-adsorbent drastically reduces the overall conversion performance of the resulting NiO-based $p$-DSCs mainly because of the decrease in $J_{\text {SC. }}$ Surfaceimmobilized CDCA did not interfere with the mechanism of charge injection effectuated by the photoexcited squaraines. The present work gives the further confirmation that surface-immobilized CDCA acts as a deactivating agent of the photoelectrochemical activity of squaraine-sensitized $\mathrm{NiO}$. This was evidenced through a drastic decrease in the conversion efficiency parameters when CDCA concentration increased in the solution of sensitization. This study reports one of the few cases in which the use of the anti-aggregating CDCA does not produce any beneficial effect on the sensitizing action of squaraines DS_45, DS_47 and pSQ2. The analysis of the electrochemical impedance spectra was conducted assuming that the model of the transmission line developed by Bisquert for the $n$-DSCs was valid also for $p$-DSCs. The potential dependence of the fitting electrical parameter showed that forward bias of the $p$-DSC induces a decrease in both resistive terms $R_{\mathrm{t}}$ and $R_{\mathrm{rec}}$. Such a decrease in $R_{\mathrm{t}}$ and $R_{\mathrm{rec}}$ was common to the $p$-DSCs sensitized with all the squaraines considered here. The chemical capacitance did not show any univocal trend with the applied potential when the concentration of CDCA was varied (vide supra).

\section{ACKNOWLEDGMENTS}

The authors acknowledge the financial support from MIUR which funded the research project PRIN $2010-2011$ (protocol no. 20104XET32). D.D. acknowledges the financial support from the University of Rome "LA SAPIENZA" through the program Ateneo 2012 (Protocol No. C26A124AXX). A.D.C. thanks Regione Lazio and CHOSE for the technical support of the research conducted at the University of Rome "Tor Vergata". ADC gratefully acknowledge the financial support of the Ministry of Education and Science of theRussian Federation in the framework of Increase Competitiveness Program of NUST «MISiS» (№ K2-2017- 025), implemented by a governmental decree dated 16th of March 2013, N 211.

\section{CONFLICT OF INTEREST STATEMENT}

The authors declare that there is no conflict of interests regarding the publication of the present paper.

\section{REFERENCES}

[1] O’Regan, B. and Gratzel, M. 1991, Nature. 353, 737.

[2] Fakharuddin, A., Jose, R., Brown, T.M., Fabregat-Santiago, F. and Bisquert, J. 2014, Energy Environ. Sci. 73952. doi:10.1039/c4ee01724b.

[3] Bard, A. J. 1980, Science 207, 139.

[4] Gerischer, H., Michel-Beyerle, M.E., Rebentrost, F. and Tributsch, H. 1968, Electrochim. Acta. 13 , 1509. doi:10.1016/0013-4686(68)80076-3.

[5] Moser, J.E. in Dye-Sensitized Solar Cells; Kalyanasundaram, K., Ed.; EPFL Press: Lausanne, Switzerland, (2010) 403-456.

[6] Fujita, S. 2015, Jpn. J. Appl. Phys. 54, 30101. doi:10.7567/JJAP.54.030101.

[7] Hagfeldt, A., Boschloo, G., Sun, L., Kloo, L. and Pettersson, H. 2010, Chem. Rev. $110,6595$. doi:10.1021/cr900356p. 
[8] Ooyama, Y. and Harima, Y. 2012, ChemPhysChem. 13, 4032. doi:10.1002/cphc.201200218.

[9] Kakiage, K., Aoyama, Y., Yano, T., Oya, K., Fujisawa, J. and Hanaya, M. 2015, Chem. Commun. $51,15894$. doi:10.1039/C5CC06759F.

[10] Perera, I. R., Daeneke, T., Makuta, S., Yu, Z., Tachibana, Y., Mishra, A., Bauerle P., Ohlin C.A., Bach U. and Spiccia L. 2015, Angew. Chemie - Int. Ed. 54, 3758. doi:10.1002/anie.201409877.

[11] He, J., Lindström, H., Hagfeldt, A. and Lindquist, S. E., 2000, Sol. Energy Mater. Sol. Cells. $62,265$. http://www.sciencedirect.com/science/article/pii/S0927024899001683?via\%3Dihub

[12] Nakasa, A., Usami, H., Sumikura, S., Hasegawa, S., Koyama, T. and Suzuki, E. 2005, Chem. Lett. $34,500$. doi:10.1246/cl.2005.500.

[13] Nattestad, A., Perera, I. and Spiccia, L. 2016, J. Photochem. Photobiol. C Photochem. Rev. 2844. doi:10.1016/j.jphotochemrev.2016.06.003

[14] Congiu, M., De Marco, M. L., Bonomo, M., Dini, D. and Graeff, C. F. O. 2017, J. Nanoparticle Res. 19 , 7. https://www.springerprofessional.de/en/pristine-and-al-doped-hematite-printed-films-as-photoanodes-of$\mathrm{p} / 11959488$

[15] Shockley, W. and Queisser, H. J. 1961, J. Appl. Phys. 32, 510. doi:10.1063/1.1736034

[16] He, J., Lindström, H., Hagfeldt, A. and Lindquist, S. E. 1999, J. Phys. Chem. B, 103, 8940. doi:10.1021/jp991681r.

[17] Boschloo, G. and Hagfeldt, A. 2001, J. Phys. Chem. B. 105, 3039. doi:10.1021/jp003499s

[18] Vera, F., Schrebler, R., Muñoz, E., Suarez, C., Cury, P., Gómez, H., Cordova, R., Marotti, R. and Dalchiele E. A. 2005, Thin Solid Films, 490, 182. doi:10.1016/j.tsf.2005.04.052.

[19] Sumikura, S., Mori, S., Shimizu, S., Usami, H. and Suzuki, E. 2008, J. Photochem. Photobiol. A Chem. $199,1$. http://www.sciencedirect.com/science/article/pii/S1010603008001810?via\%3Dihub

[20] Lepleux, L., Chavillon, B., Pellegrini, Y., Blart, E., Cario, L., Jobic, S. and Odobel, F. 2009, Inorg. Chem. 48, 8245. doi:10.1021/ic900866g.

[21] Awais, M., Rahman, M., MacElroy, J. M. D., Coburn, N., Dini, D., Vos, J. G. and Dowling, D. P. 2010, Surf. Coatings Technol. 204, 2729. doi:10.1016/j.surfcoat.2010.02.027.

[22] Li, N., Gibson, E. A., Qin, P., Boschloo, G., Gorlov, M., Hagfeldt, A. and Sun, L. 2010, Adv. Mater. $22,1759$. doi:10.1002/adma.200903151.

[23] Awais, M., Rahman, M., MacElroy, J. M. D., Dini, D., Vos, J. G., Dowling, D. P. 2011, Surf. Coatings Technol. 205, S245. doi:10.1016/j.surfcoat.2011.01.020.

[24] Powar, S., Wu, Q., Weidelener, M., Nattestad, A., Hu, Z., Mishra, A., Bäuerle, P., Spiccia, L., Cheng Y. B. and Bach U., 2012, Energy Environ. Sci. 5, 8896. doi:10.1039/C2EE22127F.

[25] Zhang, X. L., Zhang, Z., Chen, D., Bäuerle, P., Bach, U. and Cheng, Y.B., 2012, Chem. Commun. $48,9885$. doi:10.1039/c2cc35018a.

[26] Gibson, E. A., Awais, M., Dini, D., Dowling, D. P., Pryce, M. T., Vos, J. G., Boschloo, G. and Hagfeldt, A., 2013, Pccp. 15, 2411. doi:10.1039/c2cp43592f

[27] Awais, M., Gibson, E. A., Vos, J. G., Dowling, D. P., Hagfeldt, A. and Dini, D., 2014, ChemElectroChem. 1, 384. doi:10.1002/celc.201300178.

[28] Flynn, C. J., Oh, E. E., McCullough, S. M., Call, R. W., Donley, C. L., Lopez, R. and Cahoon J. F., 2014, J. Phys. Chem. C. 118, 14177. doi:10.1021/jp5027916.

[29] D’Amario, L., Boschloo, G., Hagfeldt, A. and Hammarström L., 2014, J. Phys. Chem. C. $118,19556$. doi:10.1021/jp504551v.

[30] Naponiello, G., Venditti, I., Zardetto, V., Saccone, D., Di Carlo, A., Fratoddi, I., Barolo, C. and Dini, D., 2015, Appl. Surf. Sci. 356, 911. doi:10.1016/j.apsusc.2015.08.171.

[31] Wood, C. J., Summers, G. H., Clark, C. A., Kaeffer, N., Braeutigam, M., Carbone, L. R., D’Amario, L., Fan, K., Farré, Y., Narbey, S., Oswald, F., Stevens, L. A., Parmenter, C. D. J., Fay, M. W., La Torre, A., Snape, C. E., Dietzek, B., Dini, D., Hammarstrom, L., Pellegrin, Y., Odobel, F., Sun, L., Artero, V. and Gibson, E. A., 2016, Phys. Chem. Chem. Phys. 18, 10727. doi:10.1039/c5cp05326a.

[32] Bonomo, M., Naponiello, G., Venditti, I., Zardetto, V., Di Carlo, A. and Dini, D., 2017, J. Electrochem. Soc. 164, H137. doi:10.1149/2.0051704jes.

[33] Mizoguchi, Y. and Fujihara, S., 2008, Electrochem. Solid-State Lett. 11, K78. doi:10.1149/1.2929665.

[34] Powar, S., Daeneke, T., Ma, M. T., Fu, D., Duffy, N. W., Gotz, G., Weidelener, M., Mishra, A., Bauerle, P., Spiccia, L. and Bach, U., 2013, Angew. Chemie - Int. Ed. 52, 602. doi:10.1002/anie.201206219.

[35] Powar, S., Bhargava, P., Daeneke, T., Götz, G., Bäuerle, P., Geiger, T., Kuster, S., Nuesch, F., Spiccia, L. and Bach, U., 2015, Electrochim. Acta. 182, 458. doi:10.1016/j.electacta.2015.09.026.

[36] Gorlov, M. and Kloo, L., 2008, Dalt. Trans., 2655. doi:10.1039/b716419j.

[37] Nattestad, A., Mozer, A. J., Fischer, M. K. R., Cheng, Y. B., Mishra, A., Bauerle, P. and Bach, U. 2010, Nat Mater. 9,31 .

[38] Lefebvre, J. F., Sun, X. Z., Calladine, J. A., George, M. W. and Gibson E. A., 2014, Chem. Commun. $50,5258$. doi: $10.1039 / \mathrm{c} 3 \mathrm{cc} 46133 \mathrm{e}$.

[39] Wood, C. J., Summers, G. H. and Gibson, E. A.; 2015, Chem. Commun. 51, 3915. doi:10.1039/c4cc10230d.

[40] Bonomo, M., Sabuzi, F., Di Carlo, A., Conte, V., Dini, D. and P. Galloni, 2017, New J. Chem. $41,2769$. 
doi:10.1039/C6NJ03466G.

[41] Bonomo, M., Saccone, D., Magistris, C., Di Carlo, A., Barolo, C. and Dini, D., 2017, ChemElectroChem doi:10.1002/celc. 201700191

[42] Law, K. K. and Bailey, F. C. 1992, J. Org. Chem. 57, 3278. http://pubs.acs.org/doi/abs/10.1021/jo00038a010.

[43] Shi, Y., Hill, R. B. M., Yum, J. H., Dualeh, A., Barlow, S., Grätzel, M., Marder, S. R. and Nazeeruddin, M. K., 2011, Angew. Chemie - Int. Ed. 50, 6619. doi:10.1002/anie.201101362.

[44] Park, J., Barolo, C., Sauvage, F., Barbero, N., Benzi, C., Quagliotto, P., Coluccia S., Di Censo, D., Graetzel, M., Nazeeruddin M. K. and Viscardi, G., 2012, Chem. Commun. 48, 2782. doi:10.1039/c2cc17187b.

[45] Park, J., Barbero, N., Yoon, J., Dell’Orto, E., Galliano, S., Borrelli, R., Hum, J. H., Di Censo, D., Graetzel, M., Nazeeruddin M. K., Barolo, C. and Viscardi, G., 2014, Phys. Chem. Chem. Phys. 16, 24173. doi:10.1039/c4cp04345f.

[46] Galliano, S., Novelli, V., Barbero, N., Smarra, A., Viscardi, G., Borrelli, R., Sauvage, F. and Barolo, C., 2016, Energies, 9, 486. doi:10.3390/en9070486.

[47] Venditti, I., Barbero, N., Russo, M.V., Di Carlo, A., Decker, F., Fratoddi, I., Barolo, C. and Dini, D., 2014, Mater. Res. Express. 1, 15040. doi:10.1088/2053-1591/1/1/015040.

[48] Chen, G., Sasabe, H., Igarashi, S., Hong, Z. and J. Kido, 2015, J. Mater. Chem. A. 3 , 14517. doi:10.1039/C5TA01879J.

[49] Funabiki, K., Mase, H., Saito, Y., Otsuka, A., Hibino, A., Tanaka, N., Miura, H., Himori, Y., Yoshida, T., Kubota, Y. and Matsui, M., 2012, Org. Lett. 14, 1246. doi:10.1021/ol300054a.

[50] Barbero, N., Magistris, C., Park, Y., Saccone, D., Quagliotto, P., Buscaino, R., Medana, C., Barolo, C. and Viscardi, G. 2015, Org. Lett. 17, 3306. doi:10.1021/acs.orglett.5b01453.

[51] Park, J., Viscardi, G., Barolo, C. and Barbero, N., 2013, Chimia (Aarau). 67, 129. doi:10.2533/chimia.2013.129.

[52] Magistris, C., Martiniani, S., Barbero, N., Park, J., Benzi, C., Anderson, A., Law C., Barolo, C. and O’Regan B., 2013, Renew. Energy, 60, 672. doi:10.1016/j.renene.2013.06.018.

[53] Yang, D., Sasabe, H., Jiao, Y., Zhuang, T. and Huang, Y., 2016 Mater. Chem. A, 4, 18931. doi:10.1039/C6TA08684E.

[54] Rao, G.H., Venkateswararao, A., Giribabu, L., and Singh, S.P., 2016, Photochem. Photobiol. Sci. $15,287$. doi:10.1039/C5PP00335K.

[55] Bonomo, M., Barbero, N., Matteocci, F., Di Carlo, A., Barolo, C. and Dini, D., 2016, J. Phys. Chem. C. 120, 16340. doi:10.1021/acs.jpcc.6b03965.

[56] Chang, C. H., Chen, Y. C., Hsu, C. Y., Chou, H. H. and Lin, J. T., 2012, Org. Lett. 14 , 4726. doi:10.1021/ol301860w.

[57] Warnan, J., Gardner, J., Le Pleux, L., Petersson, J., Pellegrin, Y., Blart, E., Hammarstrom L. and Odobel, F., 2014, J. Phys. Chem. C. 118, 103. doi:10.1021/jp408900x.

[58] Yen, Y. S., Chen, W. T., Hsu,C. Y., Chou, H. H., Lin J. T. and Yeh, M. C. P., 2011, Org. Lett. 13 , 4930. doi:10.1021/ol202014x.

[59] Yum, J. H., Moon, S. J., Humphry-Baker, R., Walter, P., Geiger, T., Nüesch, F., Graetzel, M. and Nazeeruddin, M.K., 2008, Nanotechnology, 19, 424005. doi:10.1088/0957-4484/19/42/424005.

[60] Neale, N. R., Kopidakis, N., Van De Lagemaat, J., Grätzel, M. and Frank, A. J., 2005, J. Phys. Chem. B. 109, 23183. doi:10.1021/jp0538666.

[61] Manthou, V. S., Pefkianakis, E. K., Falaras, P. and Vougioukalakis G.C., 2015, ChemSusChem. 8 , 588. doi:10.1002/cssc.201403211.

[62] Oscarsson, J., Hahlin, M., Johansson, E. M .J., Eriksson, S. K., Lindblad, R., Eriksson, A. I. K., Zia, A., Siegbahn H. and Rensmo H., 2016, J. Phys. Chem. C. 120, 12484. doi:10.1021/acs.jpcc.6b02521.

[63] Salvatori, P., Marotta, G., Cinti, A., Anselmi, C., Mosconi, E. and De Angelis, F., 2013, J. Phys. Chem. C. 117, 3874. doi:10.1021/jp4003577.

[64] Xu, D., Shi, C., Wang, L., Qiu, L., and Yan, F., 2014, J. Mater. Chem. A. 2, 9803. doi:10.1039/C4TA01255K.

[65] Jiang, X., Marinado, T., Gabrielsson, E., Hagberg, D. P., Sun, L. and Hagfeldt, A., 2010, J. Phys. Chem. C. 114, 2799. doi:10.1021/jp908552t.

[66] Wan, Z., Jia, C., Wang, Y. and Yao, X., 2015, RSC Adv. 5 50813. doi:10.1039/C5RA06774J.

[67] Cisneros, R., Beley, M. and Lapicque, F., 2016, Phys. Chem. Chem. Phys. 18, 9645. doi:10.1039/C6CP00077K.

[68] Konstantakou, M., Falaras, P. and Stergiopoulos, T., 2014, Polyhedron. 82, 109. doi:10.1016/j.poly.2014.05.011.

[69] Alagumalai, A., Munnavar, F. M. K., Vellimalai, P., Sil, M. C. and Nithyanandhan, J., 2016, ACS Appl. Mater. Interfaces. 8, 35353. doi:10.1021/acsami.6b12730.

[70] Chen, G., Sasabe, H., Sasaki, Y., Katagiri, H., Wang, X. F., Sano, T., Hong, Z., Yang, Y. and Kido, J., 2014, Chem. Mater. 26, 1356. doi:10.1021/cm4034929.

[71] Bonomo, M., Naponiello, G., Di Carlo, A. and Dini, D., 2016, J Mater Sci Nanotechnol. 4, 201. doi: 10.15744/2348-9812.4.201

[72] De Rossi, F., Di Gaspare, L., Reale, A., Di Carlo, A. and Brown, T. M., 2013, J. Mater. Chem. A. 1 , 12941. doi:10.1039/c3ta13076b.

[73] Maeda, T., Mineta, S., Fujiwara, H., Nakao, H., Yagi, S. and Nakazumi, H., 2013, J. Mater. Chem. A, $1,1303$. 
doi:10.1039/c2ta00883a.

[74] Paek, S., Choi, H., Kim, C., Cho, N., So, S., Song, K., Nazeeruddin, M. K. and Ko, J., 2011, Chem. Commun. 47, 2874. doi:10.1039/c0cc05378c.

[75] Lee, Y. H., Kang, J. Y., Kim, D. W., Kim, J. S. and Kim, J. H., 2016, Mol. Cryst. Liq. Cryst. 635, 148. doi:10.1080/15421406.2016.1200400.

[76] Fang, M., Li, H., Li, Q. and Li, Z., 2016, RSC Adv. 6, 40750. doi:10.1039/C6RA03694E.

[77] Yum, J. H., Walter, P., Huber, S., Rentsch, D., Geiger, T., Nüesch, F., De Angelis, F., Graetzel, M. and Nazeeruddin, M. K., 2007, J. Am. Chem. Soc. 129, 10320. doi:10.1021/ja0731470.

[78] Geiger, T., Kuster, S., Yum, J. H., Moon, S. J., Nazeeruddin, M. K., Grätzel, M. and Nuesch, F., 2009, Adv. Funct. Mater. 19, 2720. doi:10.1002/adfm.200900231.

[79] Sreejith, S., Carol, P., Chithra, P. and Ajayaghosh, A., 2008, J. Mater. Chem. 18, 264. doi:10.1039/b707734c.

[80] Sheehan, S., Naponiello, G., Odobel, F., Dowling, D.P., Di Carlo, A. and Dini, D., 2015, J. Solid State Electrochem. 19, 975. doi:10.1007/s10008-014-2703-9.

[81] Bisquert, J., 2002, J. Phys. Chem. B. 106, 325. doi:10.1021/jp011941g.

[82] Bisquert, J., Garcia-Belmontea, G., Fabregat-Santiago, F. and Compte A., 1999, Electrochemistry Commun. 1, 429. doi:S1388-2481(99)00084-3.

[83] Bisquert, J., 2000, Phys. Chem. Chem. Phys. 2, 4185. doi:10.1039/b001708f.

[84] Bisquert, J., 2003, Phys. Chem. Chem. Phys. 5, 5360. doi:10.1039/b310907k.

[85] Fabregat-Santiago, F., Randriamahazaka, H., Zaban, A., Garcia-Cañadas, J., Garcia-Belmonte, G. and Bisquert, J., 2006, Phys. Chem. Chem. Phys. 8, 1827. doi:10.1039/b600452k.

[86] Jamnik, J. and Maier, J., 2001, Phys. Chem. Chem. Phys. 3, 1668. doi:10.1039/b100180i.

[87] Hagfeldt, A., Cappel, U. B., Boschloo, G., Sun, L., Kloo, L., Pettersson, H. and Gibson, E. A., in Dye sensitized photoelectrochemical cells in Practical Handbook of Photovoltaics: Fundamentals and Applications, 2nd Ed.; McEvoy, A., Markvart, T., Castaner, L., Eds.; Elsevier: Amsterdam, (2012) 479-542.

[88] Peter, L. M. and Wijayantha, U. K. G., 1999, Electrochem. Commun. 1, 576. https://doi.org/10.1016/S13882481(99)00120-4

[89] Hagfeldt, A. and Peter, L., Characterization and modeling of dye-sensitized solar cells: a toolbox approach. In DyeSensitized Solar Cells; Kalyanasundaram, K., Ed.; EPFL Press: Lausanne, Switzerland, 2010.

[90] Hagfeld, A. and Grätzel, M., 1995, Chem. Rev. 95, 49. doi: 10.1021/cr00033a003

[91] Pumiglia, D., Giustini, M., Dini, D., Decker, F., Lanuti, A., Mastroianni, S., Veyres, S. and Caprioli, F., 2014, ChemElectroChem. 1, 1388. doi:10.1002/celc.201402027. 